\title{
Incentive contrast: A review of behavioral changes following shifts in reward
}

\author{
CHARLES F. FLAHERTY \\ Rutgers University, New Brunswick, New Jersey
}

\begin{abstract}
The literature relevant to incentive contrast effects is reviewed, with emphasis on the data published since the reviews by Black (1968) and Dunham (1968). Contrary to the evidence available for the earlier reviews, the current literature indicates that positive contrast is a reliable phenomenon. Its occurrence is facilitated by use of a constant delay of reward, use of a long runway, or possibly by a shift while a negative contrast effect, resulting from a previous shift, is still present in the animals' behavior. Positive contrast also occurs in consummatory behavior when sucrose or saccharin solutions are shifted. Conditions that are ineffective in producing positive contrast are reviewed, as are the effects of numerous variables on both successive and simultaneous contrast. In addition, positive and negative contrast effects resulting from shifts in delay or percentage of reward, contrast resulting from shifts in sucrose, saccharin, or ethanol solutions, contrast in choice behavior, and transsituational contrast are reviewed. The relationship of the data to several theoretical interpretations of contrast is also considered.
\end{abstract}

Interpretation of the behavior changes that occur when a familiar reward is replaced by a novel, usually less preferred, reward has played an important role in the theoretical analysis of animal learning. For example, Tolman (1932) cited the behavior of a monkey when the familiar reward of a banana was replaced by a piece of lettuce as evidence favoring his view that animals learn expectancies of particular rewards in a learning situation. The behavioral description was obtained in a delayed-response experiment by Tinklepaugh (1928): "She [the monkey] extends her hand to seize the food. But her hand drops to the floor without touching it. She looks at the lettuce but (unless very hungry) does not touch it. She looks under and around her. She picks the cup up and examines it thoroughly inside and out. She has on occasion turned toward observers present in the room and shrieked at them in apparent anger. After several seconds spent searching, she gives a glance toward the other cup, which she has been taught not to look into, and then walks off to a nearby window. The lettuce is left untouched on the floor" (p. 224).

In addition to supporting Tolman's expectancy interpretation of animal learning, this description contains elements that continue to be relevant as possible explanations of the behavioral changes that

Appreciation is due Alexandra Avdzej for assistance in library research and to Howard Becker, Susan Checke, and Mary Flaherty for their comments on the manuscript. Preparation for this article was aided by grants from the Rutgers Research Council. The author's mailing address is: Psychology Department, Busch Campus, Rutgers University, New Brunswick, New Jersey 08903. occur subsequent to reward shifts, that is, the apparent presence of an emotional response elicited by the shift and the possibility that the animals search for the missing reward, given the opportunity.

A rat study by Elliott (1928) produced a similar interpretation. In that study, rats shifted from a bran mash to a sunflower seed reward in a complex maze ran slower and entered more blind alleys than did an unshifted sunflower seed group. Elliott's interpretation of these results was that the shifted animals were searching for the missing bran mash, an interpretation consonant with Tolman's expectancy theory.

The reward-shift study that is most ws.ll known and that had the greatest impact on theory was published by Crespi (1942). Crespi's study was complicated, but the basic results are clear. That is, rats shifted from a larger to a smaller reward in a runway showed an abrupt decrement in running speed, to a level below a speed extrapolated from unshifted control animals, and, conversely, rats shifted from a smaller to a larger reward showed a rapid increase in running speed, to a level above the extrapolated level of unshifted large-reward animals. Crespi attributed the occurrence of these depression and elation effects, as he called them, to emotional responses (anger or frustration and joy) which interfered with or facilitated approach behavior.

The abruptness of the behavior change produced by the reward shifts in Crespi's experiment forced a change in Hull's interpretation (Hull, 1943) of how reward magnitude influenced behavior-a change away from the Thorndikian position, that different amounts of reward led to different habit strengths, to the idea that different amounts of reward influ- 
enced incentive motivation (Hull, 1952). Hull's switch to an incentive motivation interpretation was consistent with Crespi's theory that different amounts of reward produced different levels of "eagerness" or "anticipation" and with both Crespi's and Tolman's position that reward influenced "performance" rather than learning (in Hull's habit strength sense).

The position adopted by Hull, and more fully elaborated by Spence (1956), differed from the cognitive formulations of Tolman and Crespi in that incentive motivation was itself thought to be based on a type of S-R learning, that is, fractional anticipatory goal responses formed on the basis of Pavlovian contingencies existing in the context of the instrumental learning task.

This theoretical alteration dealt with the abruptness of behavior change produced by the reward shift, but it did not deal with the apparent reward relativity effects demonstrated by Crespi. That is, the reward shifts led not only to abrupt changes in behavior but also to changes that led to the "overshooting" or "undershooting" of the level of performance of the relevant control groups. These overshooting and undershooting (elation and depression) effects indicated that the influence exerted by a particular reward on performance depended not only on some physical characteristic of that reward (e.g., weight) but also on the animal's prior history of reward. Thus, in the postshift phase of Crespi's experiment, several groups were all receiving the same reward, but their running speeds were all different, varying inversely with the preshift level of reward.

Spence's approach to this aspect of Crespi's data was similar to that taken by Crespi himself. That is, Spence (1956) assumed that a decrease in reward led to an emotional response of frustration, components of which interfered with approach to the goalbox. This interference produced the depression effect. As for the elation effect, Spence argued that it did not exist per se. Rather, Crespi's demonstration of an apparent elation effect was due, argued Spence, to the failure to use an unshifted control group that was continued in testing throughout the postshift period (Crespi extrapolated speeds from the unshifted control animals which were terminated at the end of the preshift phase). Thus, the animals shifted upward in reward only appeared to overshoot the level of the control animals because the extrapolated curve did not represent animals running at their asymptotic speed. Spence himself provided evidence indicating that an elation effect did not occur if the appropriate controls were included.

Two major reviews of reward-shift studies published in 1968 (Black, 1968; Dunham, 1968) generally supported Spence's conclusions. That is, the literature to that time provided scant evidence of the ela- tion effect in runway studies, but substantial support for the depression outcome of reward shift. This pattern of data had implications for the theoretical interpretations of reward-shift effects in that positions implying symmetrical reward relativity effects, such as theory derived from perceptual adaptation level, were not favored, whereas theoretical positions implying interfering effects of emotional responses and/or associative generalization decrement (due to changed reward conditions) were favored.

The plan of the present paper is to review the contrast research, with emphasis on that published since the Black and Dunham articles and to consider the theoretical developments suggested by the data. The extensive data now available generate categories that were not necessary in the earlier reviews. For example, it will be useful to discuss shifts in delay of reward, as well as percentage of reward and reward magnitude, and to consider differences obtained in consummatory and instrumental tasks with sucrose rewards.

\section{Terminology}

Since the studies of Bower (1961), Spear and Spitzner (1966), and Zeaman (1949), there has been some general, but by no means complete, agreement as to the terminology applied to the various reward relativity paradigms. In particular, the term "contrast" is most frequently applied to demonstrations of reward relativity. This term was apparently carried over from perceptual research, in which it had been well established that the context in which stimuli were sensed could exert a pronounced influence on the perception of those stimuli. In particular, contrast refers to an exaggeration of apparent stimulus differences produced by close juxtaposition of two or more stimuli (Helson, 1964; Woodworth \& Schlosberg, 1954; Zeaman, 1949). In learning studies, then, contrast refers to an apparent exaggeration of reward differences brought about by animals experiencing two rewards in close temporal proximity.

In the Crespi (1942) experiment, the rats first received one level of reward and then were shifted to a different reward. This procedure will be called "successive contrast," and the depression effect reported by Crespi, a "successive negative contrast effect" (SucNCE). The elation effect will be designated a "successive positive contrast effect" (SucPCE).

Another type of contrast obtained in runway research was demonstrated by Bower (1961). Bower trained a group of rats in a differential conditioning study with a large reward correlated with one alley brightness (e.g., black) and a small reward correlated with a different alley brightness (e.g., white). When the running speed of these animals was compared with that of animals that received only a single level of reward in each alley, another example of contrast 
was obtained; the rats that received both large and small reward ran slower for the small reward than did rats that received only the small reward. This result, apparently analogous to Crespi's depression effect, has been termed a "simultaneous negative contrast effect" (SimNCE). A result showing that rats receiving both levels of reward run faster for the large reward than do animals that receive only the large rewards (a result analogous to Crespi's elation effect) would be termed a "simultaneous positive contrast effect" (SimPCE). Bower found no evidence for such a result, but some other studies (to be considered below) have.

Thus, the terms that we shall use in referring to the discrete-trials contrast literature are SucNCE, SucPCE, SimNCE, and SimPCE. Using the term "simultaneous" to refer to the Bower paradigm is somewhat misleading because the animals do not receive exposure to two cues simultaneously. In fact, Dunham (1968) suggested the term "successive differential contrast" for this paradigm (and "successive nondifferential" for the Crespi procedure), a term that is more descriptive of the actual procedure. However, since the simultaneous label seems to be most frequently used, we will continue to use that designation (cf, Spear \& Spitzner, 1966).

\section{POSITIVE CONTRAST}

In this section, evidence relevant to the existence of positive contrast in the discrete-trials procedure will be considered. The great majority of experiments reviewed were conducted in runways, used rats as subjects, and varied quantity of solid food reward. Exceptions to these generalizations will be noted when appropriate.

Despite the early demonstrations of SucPC by both Crespi (1942) and Zeaman (1949), the weight of the evidence has indicated that positive contrast either did not exist or was a much more elusive effect than negative contrast (Black, 1968; Cox, 1975; Dunham, 1968). As indicated above, Spence had argued that the early demonstrations of SucPC were artifacts of a failure to adequately consider the asymptotic running speeds of the positive contrast control group. Spence's approach was to increase the number of trials prior to a shift, thereby allowing animals to more closely approach asymptotic running speeds. When this experiment was done, Spence (1956) found a SucNCE but no SucPCE, a result supporting his argument.

However, as the negative evidence regarding the existence of SucPCE accumulated, there was also a realization that Spence's control condition might, in fact, obscure the occurrence of positive contrast. If subjects in a large-reward control group are already running as fast as they can in the apparatus, then there will be no room for animals upshifted in reward to show a PCE. This possibility became known as the "ceiling effect" problem.

One procedure used to avoid potential ceiling effects was, paradoxically, in the light of Spence's argument, to shift after a few acquisition trials, before the animals approached asymptote. This procedure was adopted by Schrier (1967), who shifted rats from 1 to 4 pellets after only 17 runway trials, an acquisition period comparable to Crespi's. However, no SucPCEs were evident in the 15 additional trials. Similar experiments by Mellgren (1971b), who shifted from 1 to 5 pellets after 0,2 , or 4 trials, by Campbell, Crumbaugh, Knouse, and Snodgrass (1970), who shifted from 2 to 24 pellets after $0,5,10$, or 15 trials, and by Ashida and Birch (1964), who shifted from 1 to 10 pellets after $0,10,20$, or 30 trials, also failed to find a SucPCE.

A second, and apparently more successful, procedure for avoiding ceiling effects is to introduce a delay of reward as a device for reducing the animals' speed. One method of using delay for this purpose was developed by Shanab, Sanders, and Premack (1969), who combined a shift from no delay to 30-sec delay of reward in a runway with an increase in amount of reward. The introduction of the delay caused all animals to run slower, but the animals shifted from a small to a large reward slowed their running less than did the animals shifted from a small to a medium reward, who, in turn, slowed their running less than did the animals maintained at the small reward. These results were regarded as demonstrating positive contrast by the authors. Another way of using delay to obviate ceiling effects is exemplified in a study by Mellgren (1972). Mellgren trained rats given either 2- or 22-pellet rewards with a constant 20-sec delay from the beginning of the experiment. In the shift phase, when half of each reward group was shifted to the opposite reward, both SucPCE and SucNCE were obtained. A number of other studies have also found SucPCEs when a delay of reward was used with either Mellgren's or Shanab's procedure (e.g., Lehr, 1974; Mellgren, 1971a; Mellgren, Seybert, Wrather, \& Dyck, 1973; Shanab \& Biller, 1972; Shanab \& Cavallaro, 1975; Shanab \& Spencer, 1978; Spencer \& Shanab, 1979). The Spencer and Shanab (1979) paper involved shifts in the magnitude of water reward under either delayed or nondelayed conditions. Both SucNCEs and SucPCEs were found when there was a 15-sec delay; neither contrast was found under no-delay conditions. The use of a delay does not, however, guarantee a SucPCE (Shanab \& McCuistion, 1970).

Another suggestion related to the ceiling effects hypothesis concerns deprivation level. Rats run more slowly in a runway under conditions of relative satiety. Therefore, it is conceivable that relatively nondeprived rats could be used in positive contrast studies to obviate ceiling difficulties. The data concern- 
ing this idea are relatively scanty and what do exist do not support the hypothesis. For example, Ehrenfreund and Badia (1962) found evidence of both SucPCEs and SucNCEs in rats deprived to $85 \%$ of their free-feeding weights but neither effect in animals at the $95 \%$ level. Two unusual aspects of this study should be mentioned. First, no control groups were included, so the estimate of contrast was based on preshift levels of running speed. However, since a large number of preshift trials were given (90), there is reason to think that these preshift speed levels constitute a stable baseline. The second feature of the study is that the trials were highly massed: all 115 trials of the pre- and postshift period were given over a 4-day period. Trials were given in blocks of five with a 15- to 20-sec intertrial interval (ITI) and with from $1 / 2$ to $1 \mathrm{~h}$ between blocks within a day. It is quite likely that massed trials favor the occurrence of contrast (see below). In another study, Ehrenfreund (1971) found no SucPC with animals at either $85 \%$ or $98 \%$ body weight, although the tendency towards PC was numerically greater in the $85 \%$ group. Shanab and Ferrell (1970) found SucPC both in rats shifted from more extreme to less extreme deprivation and at the same time given the upshift in reward and in rats maintained at the greater deprivation condition. This latter study was conducted in a complex maze, a condition which itself might serve to obviate ceiling effects problems. Finally, Benefield, Oscos, and Ehrenfreund (1974) found SucPCEs in rats at $90 \%$ and $98 \%$ ad-lib weight. In summary, the idea that SucPCE would be more likely to occur if the animals were relatively satiated is not supported, at least not by the runway data.

Another procedural detail related to the Crespi experiment, and possibly related to PCEs, is the total reward history of the animals. In Crespi's study, the rats that were upshifted in reward actually received a double shift: they were initially trained with a large reward, then shifted to a small reward and then back to a large reward. The role of prior large-reward experience has been investigated in a number of multiple-shift studies. Benefield et al. (1974) and Calef (1972) both report SucPCEs in rats that had had prior experience with the large reward. However, no direct comparison with animals not receiving such experience was made in either study. In contrast, E. J. Capaldi and Lynch (1967) and Weinstock (1971) both reported no SucPCEs in rats that had experienced a large-small-large sequence. Weinstock did find a SucPCE in animals that had experienced only a small-to-large shift.

The contradictions of these studies are not quite resolved by five other studies of repeated shifts. Two of these were runway studies with food reward. McCain and Cooney (1975) found a SucPCE under three conditions: on the first shift from small to large, also after large-small-large experience, and, finally, after small-large-small-large experience. The authors of this study questioned the validity of the contrast effect that occurred without prior largereward experience (first shift), but it was statistically reliable and the present reviewer sees no reason to treat its occurrence differently from other instances of SucPC. The second study (Maxwell, Calef, Murray, Shepard, \& Norville, 1976) was interesting in that the two groups of rats were shifted from large to small reward and then back to large after differential postshift experience with the SucNCE that resulted from the first shift. Animals that were shifted back to large reward while a SucNCE was still evident in their behavior showed a SucPCE. However, animals that were shifted back to large reward after the SucNCE had dissipated did not show a SucPCE when returned to the large reward.

Three studies remain to be mentioned. Shanab and Spencer (1978) reported SucPC in both small-large and large-small-large animals in a study in which magnitude of water reward was varied under a constant 20-sec delay. In another study, varying magnitude of sucrose with thirsty rats, a SucPCE was obtained in LSL animals, but not in SSL animals (Shanab, France, \& Young, 1976; cf., also, Shanab, Young, \& France, 1975). Finally, in a barpress experiment, Shanab, Domino, and Ralph (1978) reported no SucPCE in a small-large shift, although one did occur in a second shift to large reward after an intervening small-reward shift.

In summary, the results of four of these studies (E. J. Capaldi \& Lynch, 1967; Maxwell et al., 1976; Shanab et al., 1978; Weinstock, 1971) indicate that prior large-reward experience is not a sufficient condition for production of a SucPCE. Given the number of studies that have obtained SucPCEs without exposing the animals to prior large-reward experience, it appears that such experience is not a necessary condition for the occurrence of contrast. However, the results obtained by Maxwell et al. (1976) deserve further exploration. The authors suggest that the occurrence of SucPCEs under constant delay conditions and the occurrence of a SucPCE if a shift is made while the animals are experiencing a SucNCE may be related. They may be related because both delay and negative contrast may be considered aversive motivational states (Daly, 1974a, 1974b). In this regard, it is also interesting to note that an NCE may be enhanced under conditions of constant delay (Moore \& McHose, 1975; Spencer \& Shanab, 1979). This result may imply the additivity of two aversive conditions.

One final procedural consideration related to SucPC remains to be mentioned. Crespi used a very long runway (20 ft). Most subsequent studies have used much shorter runways. It is possible that the length of the runway itself serves to minimize ceiling effects and therefore allow for the occurrence of PC. 
In an investigation of this possibility, Seybert and Mellgren (1972) shifted rats from 1 to 8 pellets after 28 trials (4/day). Half of the animals were tested in a $113.75-\mathrm{cm}$ straight runway and half in a $326.75-\mathrm{cm}$ U-shaped runway. Only the animals in the long runway showed a SucPCE.

In addition to the runway data discussed above, SucPCEs have also been obtained in a variety of other situations: in leverpressing (e.g., Marx, 1969; Pieper \& Marx, 1963), in consummatory behavior (Lombardi, 1978; Panksepp \& Trowill, 1971; Premack \& Hillix, 1962), with brain stimulations as reward (Atrens, VonVietinghoff-Riesch \& Der-Karabetian, 1973; Panksepp \& Trowill, 1969, 1970), and with shifts in partial reward or varied reward (see below). Thus, in contrast to earlier reviews, it may be concluded that SucPCEs are, in fact, reliably obtained. Their occurrence is enhanced by constant delay of reward, possibly by long runways, and by shifting to large reward while a SucNCE is apparent in the animal's behavior. Shifting after few acquisition trials or giving prior experience with the large reward are ineffective in producing SucPCE.

SimPCEs have remained more elusive than SucPCEs. In differential conditioning studies, the results obtained by Bower (1961) still seem to be the rule, that is, SimNCE but no SimPCE. SimNCEs were obtained in 24 of the 27 rat studies reviewed. In addition, SimNCEs have been obtained in a number of fish and turtle studies (see below-Contrast from a Comparative Perspective). However, SimPCEs have clearly occurred in only 7 standard differential conditioning studies to be reviewed below. Of studies that have included controls for both types of contrast, only 3 have found both, whereas 9 have found only negative contrast. In fact, many studies find the opposite of SimPC, a reliable depression in S+ speeds of a differential conditioning group as compared with a large-reward control group (Bower, 1961; Chechile \& Fowler, 1973; Calef, Calef, Maxwell, \& McHewitt, 1975, Experiment 2; McHewitt, 1974; Matsumoto, 1969).

Despite the numerical evidence, it is not reasonable to conclude that those few studies showing a SimPCE represent chance findings. Several of the studies that have obtained SimPCE have included special conditions designed to maximize the likelihood of such an occurrence. For example, as in the case of SucPCEs, training the animals with a constant 20-sec delay of reward seems to lead to SimPCE, perhaps because of the removal of a ceiling restraint (Calef et al., 1975; Mellgren, Wrather, \& Dyck, 1972). In fact, Calef et al. found a SimPCE in Experiment 1, in which the delay (plus low deprivation) was employed, and the opposite of a SimPCE (S+ depression) in Experiment 2, in which no delay and more extreme deprivation were used. The Mellgren et al. (1972) study also included no intermixing of $\mathrm{S}+$ and $\mathrm{S}-$ trials within a day and startboxes that were the same brightness as the alley. These two procedures were designed to reduce "decision time" on the part of the rat, time which might interfere with the measurement of SimPCEs. In a later study, Mellgren and Dyck (1974) also found both SimNCEs and SimPCEs when the startbox was the same brightness as the alley and when the large- and small-reward trials were presented in regular sequences. However, when the large- and small-reward trials were presented in a semirandom sequence, no PCE was obtained. Again, these data suggest that making the type of reward highly predictable by including redundant cues, such as alley brightness and regular reward sequences, facilitates the occurrence of SimPCE.

In one of the remaining studies that obtained a SimPCE in differential conditioning, Spear and Pavlik (1966) obtained their result with a 24-h ITI, a condition under which no SimNCE occurred. This experiment may represent a chance finding of SimPCE, since two later studies obtained a SimPCE only when the ITI between an S- and an S+ trial was made very short $(8-15 \mathrm{sec})$ and no SimPCE if the ITI was as long as $8 \mathrm{~min}$ (Fox, Calef, Gavelek, \& McHose, 1970; McHewitt, Calef, Maxwell, Meyer, \& McHose, 1969). Furthermore, the general evidence (reviewed below) indicates that contrast varies inversely with ITI.

The remaining study that obtained both a SimPCE and a SimNCE (Morrison \& Porter, 1965) used an unusual apparatus - a wedge-shaped choice chamber and a leverpress requirement. This study was also unusual in that the PCE was much more robust than the NCE. It is possible that the robustness of the PCE in this experiment is accounted for by the following considerations. The rats in this experiment received 18 trials/day split into three blocks of 6 trials each, and response speeds were measured on the last 2 trials of each block (which were forced trials). The large reward was a 97-mg pellet, and the small reward was a $20-\mathrm{mg}$ pellet. It is possible that the large-reward control group was in a greater state of "momentary satiation" as a result of these reward differences (3.88 vs. $2.34 \mathrm{~g}$ at the start of the fifth trial) and therefore that at least part of the SimPCE was due to a depression in the speed of the control group. Unfortunately, the ITI, which would be an important consideration in any "momentary satiation" interpretation, was not specified.

Another experimental device that may be relevant to this topic is the Amsel double runway (Amsel \& Roussell, 1952). Daly (1968) reasoned that when different rewards were given in the two goalboxes, the two runways essentially constituted a differential conditioning situation. Using this apparatus, Daly found evidence of a SimPCE in the sense that a group given 6 pellets in both goalbox 1 and goalbox 2 ran slower in the second alley than did a group 
given 1-pellet reward in goalbox 1 and the 6-pellet reward in goalbox 2. The occurrence of an apparent SimPCE in this apparatus without the use of delay may be related to Mellgren's idea (Mellgren, 1972) of "decision time." Since the runways were in sequence and the second goalbox always contained 6 pellets, there was no basis for uncertainty (see McHose \& Howard, 1973) to develop and interfere with the increased speed that is the indicant of the PCE.

In general, my position is to accept the existence of SimPCEs despite the numerical evidence. This leaning is based on (1) the special procedures used in the studies that provided clear evidence of SimPCEs, (2) the ready demonstration of both PCE and NCE in a consummatory response procedure that seems to be analogous to the runway differential conditioning experiment (e.g., Flaherty \& Largen, 1975, and other studies to be reviewed below when sucrose studies are considered), and (3) the abundant demonstrations of positive behavioral contrast, a paradigm that has several substantial similarities to runway differential conditioning. ${ }^{1}$

\section{VARIABLES INFLUENCING DISCRETE-TRIALS CONTRAST}

\section{Reward Disparity}

The greater the difference between large and small reward, the greater is the contrast. There is substantial evidence supporting this generalization for all but the SimPCE design. For example, DiLollo and Beez (1966) reported such a result for different groups of rats shifted from $16,8,4$, or 2 pellets to 1 pellet in the SucNCE design. Crespi $(1942,1944)$ reported "substantially more disruption of behavior" in rats shifted from 256 units of reward to 16 units than in rats shifted from 64 to 16 , and Gonzalez, Gleitman, and Bitterman (1962) reported that rats shifted from 32 to 2 pellets showed a larger SucNCE than did rats shifted from 8 to 2 pellets, and both of these groups showed larger contrast than animals shifted gradually from 32 to 2 pellets in units of 2 pellets (i.e., 32 to 30 to 28 , etc.).

In the Gonzalez et al. study, the gradually shifted group did not show a contrast. However, Daly (1974b) found a SucNCE in rats shifted gradually (1 pellet per trial) from 14 pellets to 1 pellet. In addition, Daly reported that these animals learned a hurdle-jumping task to escape from the goalbox with the reduced reward and that the speed of jumping in this group was no different from that of animals shifted abruptly from 14 pellets to 1 pellet. Daly's interpretation of these data was that both gradual and abrupt reduction of reward is frustrating (as indicated by the escape learning). The difference between the Gonzalez et al. and the Daly results in regard to the effects of a gradual shift could be due to the trial spacing (1 trial/day in Gonzalez et al. and 6/day with a 6-8-min ITI in the Daly study) and to the fact that apparently only two trials were given at the lowest reward level in the Gonzalez et al. study (because of equipment breakdown); Daly found that the NCE in her gradually shifted group appeared on the third trial after they had reached the lowest reward level. In the case of SucPC, greater contrast with greater increases in quality or quantity of reward have been reported by Marx (1969), Mellgren, Seybert, Wrather, and Dyck (1973), and Weinstein (1977). Positive contrast has also been examined in terms of increased reward, decreased delay, and increased percentage of reward (Shanab \& Cavallaro, 1975). The combination of all three factors led to greater SucPCE than did any combination of double shifts. Shifts in delay or percentage alone did not lead to SucPCE, but shift in magnitude did.

SucPCEs have also been obtained when reward was gradually increased from 1 pellet to 12 pellets (Nation, Roop, \& Dickinson, 1976). In that experiment, there was some evidence that the SucPCE was greater and more enduring in the gradual group than in the abrupt groups. There was also some evidence that the PCE with a gradual shift was more likely to occur after extended training than after limited training. It should be noted that in the Nation et al. experiment, as in the Mellgren et al. (1973) study, a constant 20-sec delay was used and that, in both experiments, the SucPCEs obtained appeared to be long lasting.

Differential conditioning studies indicate that the effect of reward disparity on SimNCE is similar to its effect on SucNCE. Davenport (1962), with a Y-maze, and Ludvigson and Gay (1966) with straight alleys, both obtained running speed to an S- reward that varied inversely as a function of the magnitude of the S+ reward. Spear and Spitzner (1966) found a similar effect when the $S-$ reward was zero (12 pellets vs. 0 , and 1 pellet vs. 0 ). None of these studies reported any tendency towards SimPCE, and neither did Matsumoto (1969) in comparisons of 25 vs. 5, 25 vs. 1 , and 5 vs. 1 (with 25-25 and 5-5 control groups). Matsumoto did, however, find numerical evidence of a greater SimNCE in 25 vs. 1 pellet animals than in 5 vs. 1 pellet groups (compared with 1-1 controls). In a related finding, Mackinnon (1968) reported more frequent retracing in the $\mathrm{S}$ - runway in rats given rewards of $500 \mathrm{mg}$ vs. 0 and $500 \mathrm{mg}$ vs. $37 \mathrm{mg}$ than in rats given rewards with smaller disparities (500 vs. $90 \mathrm{mg}, 500$ vs. $250 \mathrm{mg}$ ). Thus, in the SimCE paradigm, there is clear asymmetry in the effects of reward disparity: SimNCE varies with reward disparity; SimPCE does not. However, there have been no studies yet that have investigated the effects of reward disparity on SimPCEs using procedures that 
apparently enhance the likelihood of obtaining PCE, procedures such as those used by Calef et al. (1975) and Mellgren et al. (1972).

\section{Deprivation State}

A number of studies have indicated that a SucNCE is more likely to occur under high- than under lowdeprivation states (Cleland, Williams, \& DiLollo, 1969; Ehrenfreund, 1971; Ehrenfreund \& Badia, 1962; Flaherty \& Kelly, 1973). In these studies, the high-deprivation states included $75 \%$ and $85 \%$ ad-lib weight, and 22-h food deprivation. The less severe deprivation conditions (in which a SucNCE was not obtained) included conditions of 6-h food deprivation and $98 \%, 95 \%$, and $90 \%$ ad-lib body weights.

A study by E. D. Capaldi and Singh (1973) failed to find an influence of deprivation on SucNC. In one experiment, rats were shifted from 20 pellets to 2 pellets at either $75 \%$ or $90 \%$ ad-lib weight, and in a second experiment the shift was from 10 pellets to 1 at either $70 \%$ or $90 \%$ ad-lib weight. In both of these experiments, roughly equivalent SucNCEs occurred at both levels of deprivation. The investigators interpreted the discrepancy between their data and the earlier studies in terms of conditions optimally leading to contrast. In particular, the conditions in their experiment may have been, in one or more ways, more favorable to the occurrence of contrast than were the conditions in the earlier experiments: greater reward discrepancy (the Ehrenfreund studies), shorter intertrial interval (Cleland et al.), or more trials per day and shorter ITI (Flaherty \& Kelly). In general, Capaldi and Singh suggest that increased deprivation will enhance SucNCE under conditions that are otherwise unfavorable to the occurrence of contrast.

E. D. Capaldi has published several studies which indicate that the shift of both reward magnitude and deprivation condition concurrently prevents the occurrence of contrast (e.g., E. D. Capaldi, Smith, \& White, 1977). Capaldi's interpretation of these data is that the stimulus condition (or "state") correlated with a deprivation level is a potent cue for reward expectancy and that if this cue is changed at the same time that reward is shifted, then no contrast will occur because the preshift reward will no longer be expected.

These E. D. Capaldi studies involved SucNCEs. There are also two studies involving upward shifts in reward and concurrent deprivation shifts. E. D. Capaldi (1971) shifted from 11 to $20 \mathrm{~g}$ of food/day and increased reward from 1 to 10 pellets; no SucPCEs were obtained. Shanab and Ferrell (1970) also shifted from high- to low-deprivation state and concurrently shifted reward from 1 to 22 pellets. In this study, a SucPCE was obtained in the rats experiencing the double shift as well as in rats maintained under the more extreme deprivation conditions. There seem to be two procedural differences, one or both of which may account for the differences in results obtained between these studies. Perhaps the most important is the way in which deprivation was shifted. Capaldi gradually brought her more extremely deprived group $(11 \mathrm{~g} /$ day) up to the level of the less extreme group $(20 \mathrm{~g} /$ day $)$ by feeding them ad lib over a 10 day period and then feeding them for 4 more days at $20 \mathrm{~g} /$ day. Over this 2 -week period, the animals were not exposed to the runway. Thus, there was a 2week intershift interval. Although there are no retention studies with exactly the parameters of the Capaldi experiment, it is clear that contrast does decrease with increasing retention interval (e.g., Ciszewski \& Flaherty, 1977; Flaherty \& Lombardi, 1977; Gonzalez, Fernhoff, \& David, 1973; see also below). Shanab and Ferrell, on the other hand, shifted deprivation differently. Their extreme deprivation group was tested at $80 \%$ body weight in the first phase of the experiment; the shift of half of these animals to less extreme conditions was made by allowing them access to food for $18 \mathrm{~h}$ after each day's test session. Thus, the deprivation shift was from $80 \%$ ad-lib weight and once/day feeding to a 6-h deprivation schedule. Under these conditions, and with no time gap between shift phases, the concurrent shift in deprivation and reward did not obviate the SucPCE. The second procedural difference between these studies was that the Capaldi study was conducted in a runway, whereas the Shanab and Ferrell study was in a Lashley maze, an apparatus perhaps more conducive to SucPC because of the possible alleviation of ceiling effects.

As for the effects of stable deprivation conditions on SucPCEs, the evidence is not clear. There are studies that have obtained SucPCEs when animals were not extremely deprived (e.g., Benefield et al., 1974; Marx, 1969; Shanab \& Ferrell, 1970; Weinstock, 1971), and there are also studies that have obtained SucPCEs when animals were under more severe deprivation conditions (Crespi, 1942; Mellgren, 1971a, 1972; Mellgren et al., 1973; Shanab \& Biller, 1972; Shanab \& Cavallaro, 1975; Shanab \& Ferrell, 1970). Ehrenfreund and Badia (1962) compared upward reward shifts directly at $85 \%$ and $95 \%$ ad-lib weight and obtained SucPC only in the $85 \%$ group (but recall that this study did not have unshifted control groups; the preshift baseline was used as the control condition). Ehrenfreund (1971) found a nonsignificant tendency for SucPCEs in rats at the $85 \%$ body-weight level, but no tendency in rats at $98 \%$.

Our interpretation of all these data is that there is a tendency for degree of successive contrast to vary directly with degree of deprivation. However, this relationship may be masked by a number of procedural (e.g., apparatus, use of constant delay) and parametric (e.g., reward disparity, trials/day, ITI) 
considerations that apparently interact with the deprivation effect. The clear demonstration of the existence and nature of these presumed interactions, and thus the understanding of how deprivation influences SucCEs, awaits a heroic, tedious, expensive, and unfundable series of studies.

Systematic investigation of the effects of deprivation on SimCEs seems not to have been undertaken.

\section{Retention Interval}

The effects of retention interval, at least, are straightforward. The longer the interval between the last experience with the preshift reward and the first experience with the postshift reward, the smaller is the contrast. This, not too surprising, generalization rests on data obtained only for the SucNCE paradigm. In the runway, SucNCE resulting from a 20-to-2 pellet shift is lost with a 68-day retention interval, both when the interval is inserted between shift phases and when the interval is inserted after the first experience with the postshift reward (Gleitman \& Steinman, 1964). Also in the runway, with an 18-to-2 pellet shift, SucNC occurs with a 1-day retention interval, is smaller after $\mathbf{2 6}$ days, is numerically but not statistically present after 42 days, and is not present after 68 days (Gonzalez, Fernhoff, \& David, 1973).

A SucNCE is also obtained in consummatory behavior following a shift in the concentration of sucrose solutions from $32 \%$ to $4 \%$ (Vogel, Mikulka, \& Spear, 1968). With a 10-day preshift period (at $5 \mathrm{~min}$ access/day to the sugar solutions) contrast shows an orderly diminution as a function of an interpolated retention interval prior to a shift to $4 \%$ sucrose. With data points obtained at 1-, 4-, 5-, 10-, $17-$, and 32-day intervals, contrast is clearly present up to 10 days, is statistically marginal at 17 days, and is not present at 32 days (Ciszewski \& Flaherty, 1977; Flaherty, Capobianco, \& Hamilton, 1973; Flaherty \& Lombardi, 1977; Gordon, Flaherty, \& Riley, 1973). It has further been shown that prior discriminative experience with both the preshift and postshift solutions has an enhancing effect on the degree of contrast that occurs after a 10-day interval (Flaherty \& Lombardi, 1977), but reinstatement treatments inserted during the retention interval seem to have no effect in prolonging the memory of the preshift sucrose solution (Ciszewski \& Flaherty, 1977). It has also been shown that SucNCEs will occur if rats have only a 5-min preshift access period to $32 \%$ sucrose and then are shifted to $4 \%$ sucrose, and that this contrast effect is statistically reliable with retention intervals ranging from $20 \mathrm{~min}$ to $70 \mathrm{~h}$ (Flaherty, Ciszewski, \& Kaplan, 1979).

There seem to be no studies that have investigated retention of SucPCE or of SimCEs.

\section{Intertrial Interval}

A variable conceptually related to retention interval is intertrial interval (ITI). Contrast effects seem to vary inversely with ITI. E. J. Capaldi (1972) and Sparling and E. J. Capaldi (Note 1) have directly compared SucNCEs obtained with ITIs in the 2- to 5-min range with 24-h ITIs and found contrast to be considerably greater at the shorter ITI. There seem to be no studies that have directly examined the effects of ITI on SucPCEs.

In the simultaneous paradigm, Spear and Pavlik (1966) found a SimPCE but no SimNCE with a 24-h ITI. Since this was one of the few studies in which SimPCE had been obtained and a SimNCE had failed to occur, it appeared that ITI might systematically influence SimCEs. Spear and Spitzner (1966) followed up this study by comparing $15-\mathrm{sec}$ vs. 15-min ITIs in the context of a 12- to 1-pellet T-maze discrimination task. In this study, a SimNCE was found at both ITIs and a SimPCE at neither. Haggbloom (1979) found a SimNCE with a 3- to 4-min ITI but none with a $24-h$ ITI. Thus, it seems likely that degree of SimNCE decreases with increasing ITIs.

Again, there has been little in the way of systematic investigation of ITI in the SimPCE paradigm. In two studies, an apparent SimPCE was obtained with very short ITIs (Fox et al., 1970; McHewitt et al., 1969) but not with longer ITIs (Fox et al., 1970). In a variant of the SimCE procedures that was much like a behavioral contrast experiment, Gonzalez and Champlin (1974) found a SimPCE with a 0-sec ITI, but no reliable SimPCE with a 2-sec ITI. These results are not in agreement with the Spear and Pavlik result reported above but are consistent with the effects of ITI on other types of contrast.

A research problem, possibly related to positive contrast, is that of positive induction. Positive induction is the term used by Pavlov (1927) to describe an exaggerated conditioned response elicited by a positive conditioned stimulus (CS+) when the CS+ was intermixed with, or followed, a series of stimuli signaling the absence of reward (CS-). Thus, both induction and contrast refer to the exaggerations in behavior based on juxtaposition of rewards or on reward-signaling stimuli. In a series of experiments investigating positive induction obtained in extinction after differential conditioning (reward vs. no reward), it has been found that induction occurred when the ITI was short ( $30 \mathrm{sec}, 1 \mathrm{~min}$ ) but not when the ITI was in the 4-10-min range (Ison \& Krane, 1969; Krane \& Ison, 1970, 1971). Ison, Glass, and Daly (1969) found no evidence of positive induction in a differential conditioning study conducted with large vs. small reward (rather than reward-nonreward); however, a long ITI was used in this experiment. 
These positive-induction data are consistent with the McHewitt et al. study in indicating that SimPCEs might be facilitated by very short ITIs. Thus, although the evidence is fragmentary, most of what there is supports the idea that degree of contrast in all paradigms varies inversely with ITI.

\section{Prior Reward Experience}

Under the heading of reward disparity, it has been shown that prior (or concurrent) magnitude of reward exerts a strong influence on contrast: the greater the reward disparity, the greater the contrast. In this section, we will review the evidence concerning the effects of prior experience with the postshift reward, the effects of prior experience of partial and varied reward, the effects of specific reward sequences on contrast, and the effect of prior differential conditioning on the SucNCE.

Small reward. Some studies have shown that prior experience with the small reward in a SucNCE paradigm with a 24-h ITI eliminates contrast and with shorter ITIs substantially reduces it (E. D. Capaldi \& Singh, 1973; E. J. Capaldi, 1972; Spencer \& Shanab, 1979). A similar result has also been found in the SimCE procedure-prior experience with the S- reward reduces the degree of SimNCE (Platt \& Gay, 1968; Spear \& Spitzner, 1969a).

There are, however, studies that have shown a SucNCE in animals that have had prior experience with the small reward, and even in animals that have had several shifts (Calef, Calef, Prochaska, \& Geller, 1978; Maxwell et al., 1976; McCain \& Cooney, 1975; Shanab et al., 1978; Shanab \& Spencer, 1978). Thus, the effects of prior small-reward experience are most likely parameter-dependent. Particularly important would be intertrial interval, as E. J. Capaldi (1972) has suggested, and number of trials given in the various stages of the experiment. This latter parameter has not been systematically explored.

Partial or varied reward. SucNCE is reduced by prior experience with partial reinforcement. The initial study investigating this variable (Mikulka, Lehr, \& Pavlik, 1967) reported that prior experience with $50 \%$ reward eliminated SucNC when the animals were shifted from 10 pellets to 1 pellet (still on a $50 \%$ schedule). However, examination of the published data indicates that a numerical SucNCE did occur in the goal speed measure, although none was evident in the start measure (Mikulka et al., Figure 2). Later studies have found that prior experience with partial reinforcement seems to retard the development of contrast after a shift in reward magnitude, but that a reliable SucNCE does eventually develop (Gonzalez \& Bitterman, 1969; Ison, Glass, \& Daly, 1969; Peters \& McHose, 1974).

One study (Lehr, 1974) investigated the effect of partial reward in a SucPCE design and found that positive contrast occurred in groups shifted both from continuous and from partial 1-pellet reward to an 8-pellet reward. The schedule in effect during preshift did not alter the degree of SucPC. This study used a constant 20 -sec delay as a means of removing the ceiling restraint.

It should be noted that two different shift procedures have been used in these partial reward studies. Some of the studies maintain the partial schedule in postshift as well as in preshift (Ison et al., 1969; Mikulka et al., 1967), whereas others shift from a partial to a continuous schedule at the same time that reward is shifted (Gonzalez \& Bitterman, 1969; Lehr, 1974).

Varied magnitude of reward has many effects similar to partial reward (cf. Logan, 1960), and therefore one might expect similar effects on contrast. And, in fact, two studies have found that preshift exposure to varied reward magnitude eliminated a SucNCE when reward was shifted to constant small reward (Calef et al., 1978; Davis \& North, 1967). In a systematic study by Peters and McHose (1974), it was shown that rats given 20 and 4 pellets on a $50 \%$ schedule preshift and 1 pellet postshift showed a SucNCE roughly equivalent to that of animals receiving a constant 7-pellet reward preshift. Two other studies in this paper also showed SucNCE following varied reward experience, and the degree of contrast shown was consistent with the hypothesis that the rats "averaged" the rewards received during the preshift phase and that the degree of contrast was related to reward disparity from this average value.

The Peters and McHose study also unconfounded the effects of varied magnitude and prior experience with the postshift reward when, in two of their studies, the varied preshift reward did not include the postshift reward value. Calef, Hopkins, McHewitt, and Maxwell (1973) turned this procedure around and showed that a shift from continuous reward to varied reward also produces a SucNCE.

Finally, McHose (1970) and McHose, McHewitt, and Peters (1972) showed that partial or varied reward in $\mathrm{S}+$ of a differential conditioning study reduced the SimNCE occurring in S- (as does also reducing the proportion of S+ trials). McHose (1970) also showed that varied reward magnitude in $\mathrm{S}+$ depressed S- speeds by an amount approximately equal to that of a group given a consistent $S+$ reward whose value was near the geometric mean of the varied rewards.

Varied magnitude of reward has also been used in quite a different way in contrast research. Hulse (1973) gave three groups of rats different magazine training experiences prior to barpress acquisition. One group was trained with a constant 10-pellet reward, one group with a single-pellet reward, and the third group was given equal experience with 1 and 10 pellets. Barpress acquisition was conducted with either the single- or the 10-pellet reward. The results 
indicated that the varied-magnitude group showed a long-lasting SucNCE relative to the constant-10 group; neither group showed a SucPCE. Hulse's interpretation of the sustained contrast that occurred in the varied-magnitude group was that the prior experience of this group had established a "dimension" of reward size against which the postshift reward could be compared.

Reward sequences. E. J. Capaldi (1966) has established that specific sequences of reward and nonreward may have powerful effects in a partial reinforcement extinction experiment. The effects of some sequential variables have also been investigated in contrast experiments; however, the results have not been as clear-cut as those obtained in the extinction studies.

E. J. Capaldi and Ziff (1969) shifted two groups of rats from a $67 \%$ partial large-reward schedule to a $100 \%$ small-reward schedule. Each day, one of the partial groups received the sequence large-none-large (LNL) and the second group received the sequence LLN. There were 15 preshift trials, the large reward was $2045-\mathrm{mg}$ pellets, and the small reward was 2 45-mg pellets. A third group received only the small reward on all three daily trials throughout the experiment. After the shift, Group LLN showed a SucNCE but Group LNL did not. These results are similar to those obtained in extinction studies in which it has been found that nonreward-reward (NR) transition trials enhance resistance to extinction. Capaldi and Ziff reasoned that schedules of partial reward would retard the occurrence of SucNCE only when such NR transition trials were included in preshift experience. All of the partial-reward studies reviewed above included "random" schedules of reward and nonreward, and thus such schedules would have included NR transition trials.

Peters and McHose (1974), however, included sequential variables in their study of varied reward, and the results obtained were not consistent with a sequential analysis. Three groups of rats received 20 or 3-pellet rewards in sequences of LSL, LSS, or SSL. Following a shift to constant small reward, all groups showed a SucNCE, with contrast being largest in the LSL group, although the results Capaldi and Ziff obtained with their LNL condition would lead to the expectation that the LSL group would show the smallest contrast. Peters and McHose interpreted their results as being inconsistent with sequential theory but in agreement with an incentive averaging interpretation of varied reward (the LSL group received a larger "average" reward than the other two groups).

Of course, a major difference between the two studies just reviewed is that one included a nonreward condition whereas the other included small reward in the sequence. There has also been disagreement in the effects of sequential variables in the
SimCE paradigm and among studies that have all used large- and small-reward sequences. Campbell and Meyer (1971) examined the effects of SL and LS sequences in a differential conditioning study in which the large reward was 9 97-mg pellets and the small reward was a single such pellet. In this experiment, there were 3,4 , or 5 trials per day and Group SL experienced only small-to-large transitions within a day. Only Group LS showed a SimNCE (in comparison with a constant small-reward group). In a variation on this experiment, Meyer and Campbell (1973) found that no SimNCE occurred unless some LS transitions were included in the animals' experience (as few as one every 4 days). Group SL never showed a SimNCE. These results were obtained with both regular and irregular reward sequences.

Thus, the Campbell and Meyer studies agree with the E. J. Capaldi and Ziff SucNCE data in reward to the effectiveness of sequential variables in moderating negative contrast. However, a differential conditioning study by Mellgren and Dyck (1974) obtained results like those of the Peters and McHose SucCE study in that sequential variables were ineffective. Mellgren and Dyck used five groups of rats: one received only SL transitions, one received only LS transitions, and the third received both types of transitions; the fourth and fifth groups were contrast control groups that received only large or only small reward. The results of this study were that all groups showed a SimNCE of equivalent degree and both Group LS and Group SL showed a SimPCE (this latter result is discussed earlier in this paper). There seemed to be nothing in the procedures of these experiments that would account for such a difference in results between this experiment and the Meyer and Campbell experiments. In fact, the Mellgren and Dyck experiment used highly massed trials (15-sec ITI), a condition that should maximize the effectiveness of sequential variables. Thus, the effectiveness of sequential variables in both successive and simultaneous contrast remains unclear.

Simultaneous contrast. As a final topic in this section, we shall consider studies that have investigated the effect of prior experience with SimCE on later SucNC. The procedure used in these studies has been to shift the magnitude of reward in the S+ alley of a differential conditioning experiment or the more favorable alternative (MFA) in a choice experiment to the small reward ( $\mathrm{S}-$ reward) after a SimNCE has occurred in the S- alley. The evidence indicates that the SucNCE obtained under these conditions is smaller than a SucNCE obtained with the same reward disparity but in animals without the history of differential conditioning (Campbell \& Meyer, 1971; Ison et al., 1969; Spear and Spitzner, 1966). These results are similar to some of those obtained when animals have prior experience with the postshift re- 
ward in a SucNCE design (reviewed above), and it is not clear whether the reduction in contrast obtained in the differential conditioning studies is due to the fact that the animals have had prior experience with the small reward or due to the fact that these animals have experienced a SimNCE, or due to both of these factors.

Summary of prior reward experience. (1) The effects of prior experience with the postshift reward are unclear. Such prior experience may or may not reduce SucNCE and SimNC, possibly depending on the parameters of the experiment. (2) Prior experience with partial reward retards the development of and/or reduces the degree of SucNC. (3) Prior experience with varied reward reduces the degree of SucNCE, possibly because of an incentive averaging and reward disparity mechanism. But there may also be special procedures in which varied-magnitude experience facilitates later SucNC by establishing a "dimension" of reward magnitude. (4) The evidence concerning the effects of sequential variables on SucNC and SimNC is contradictory. (5) Prior experience with differential conditioning reduces SucNC, but the necessary and sufficient conditions for this reduction (i.e., differential conditioning or smallreward experience) are not clear.

\section{Drug and Lesion Effects}

There have been few drug studies in discrete-trials contrast. The evidence that does exist indicates that SucNCE obtained following a shift from 15 pellets to 1 pellet in the runway is eliminated by the minor tranquilizer chlordiazepoxide (Librium) in doses of 5.0 or $10.0 \mathrm{mg} / \mathrm{kg}$ but not at lower doses (Rosen \& Tessel, 1970). Chlorpromazine seems to have no effect on SucNC (Roberts \& Pixley, 1965; Rosen \& Tessel, 1970). The barbiturate amobarbital sodium eliminated a SucNCE that occurred in undrugged rats following a shift from 15 pellets to 1 pellet (Rosen, Glass, \& Ison, 1967). One study in which an attempt was made to examine the effects of amobarbital sodium on SucPCE found no definitive evidence of a SucPCE, but the drug did not hinder the rats' increase in running speed up to the levels of the controls when the reward was shifted (Ison \& Northman, 1968). There is one study that examined the effects of amylobarbitone (Ridgers \& Gray, 1973) on both negative and positive contrast, but since this study used a variety of contrast procedure that we have not considered, we must first briefly describe this paradigm. Baltzer and Weiskrantz (1970) trained rats on a variable interval (VI) 2-min schedule in which the reward was either 1 or 4 pellets. The different rewards and correlated discriminative stimuli were alternated on a daily basis. Contrast was examined by brief intrusions of alternative reward stimuli $(4.5 \mathrm{~min}$ of a $63-\mathrm{min}$ session). The results showed that intrusions of the small-reward stimulus on large-reward days led to a decrease in responding, termed a depression effect, and that intrusions of the large-reward stimulus on small-reward days led to an elevation in responding, termed an elation effect. Ridgers and Gray (1973) used this procedure and, in addition, injected the rats with $15 \mathrm{mg} / \mathrm{kg}$ of amylobarbitone at the start of a daily session. The negative contrast effect was reduced by the drug, but the positive contrast effect was uninfluenced by it.

One final study using a barbiturate (pentobarbital sodium) investigated a type of SimNCE (Brownlee \& Bitterman, 1968). This experiment was conducted with pigeons trained on a discrete-trial FR-20 keypecking response with either 8- or 1-pellet reward correlated with different stimuli. A long-lasting SimNCE occurred, and it was not influenced by pentobarbital sodium at a dosage of $5 \mathrm{mg} / \mathrm{kg}$. This dose is considerably lower than those used in the studies reporting effects of barbiturates.

In summary, chlordiazepoxide and barbiturates seem to reduce negative contrast, barbiturates may not have an effect on positive contrast, and chlorpromazine seems to have no effect on SucNCE. However, since there are so few studies and so little evidence on dose-response functions, it is not possible to make any clear statements on how the different types of contrast paradigms under discussion here are influenced by different classes of drugs. Several more drug studies will be considered below when contrast obtained in consummatory responding is considered.

There have also been few brain lesion studies conducted in connection with discrete-trials contrast. The evidence indicates that rats with the cingulate cortex removed show an attenuated SucNCE when shifted from 15 pellets to 1 in the runway (Gurowitz, Rosen, \& Tessel, 1970), and that rats with lesions of the hippocampus show no SucNCE (and no change at all) following a shift from 12 pellets to 1 in the runway (Franchina \& Brown, 1971).

A study of rats made anosmic by aspiration of the olfactory bulbs has indicated that SucNCEs occurred in both normal and operated rats, but that there was some tendency for the contrast to develop more slowly in the anosmic animals (Davis, Harper, \& Seago, 1975). There are apparently no lesion studies involving other discrete-trials contrast paradigms.

\section{SHIFTS IN DELAY OF REWARD}

The evidence concerning the occurrence of successive contrast effects with shifts in reward delay is largely negative. Five studies report a SucNCE following an increase in delay (McCain, Lobb, Almond, \& Leck, 1976; McHose \& Tauber, 1972; 
Shanab \& Biller, 1972; Shanab, Domino, \& Melrose, 1977; Shanab \& McCuistion, 1970), but eight studies failed to find a SucNCE (Ferrel \& Shanab, 1975; Harker, 1956; Logan, 1952; Mackintosh \& Lord, 1973-two experiments; McCain, Boodeé, \& Lobb, 1977; Shanab, 1971; Shanab, Rouse, \& Cavallaro, 1973). In addition, Spence (1956) reports the results of experiments by three of his students (as well as the Harker study cited above) which also failed to show evidence of a SucNCE subsequent to shifts in delay.

Examination of the parameters of these experiments failed to yield any differentiation between successes and failures in regard to any single factor. It is possible that some combination such as a large number of preshift trials, a large reward, multiple trials per day, and an intermediate delay shift (e.g., 0 to $15 \mathrm{sec}$ ) may facilitate the occurrence of contrast. However, the Mackintosh and Lord study had parameters close to these and did not obtain contrast.

In the case of SucPCEs, the lack of an effect is more convincing. There is only one instance of a SucPCE (Sgro \& Weinstock, 1963), whereas 11 studies report no SucPCE (Ferrel \& Shanab, 1975; Harker, 1956; Logan, 1952; McCain et al., 1976, 1977; McHose \& Tauber, 1972; Shanab \& Biller, 1972; Shanab \& Cavallaro, 1975; Shanab \& McCuistion, 1970; Shanab et al., 1977, 1973). The 1 study which did find the SucPCE was unusual in that water was used as the reward, there was only one trial per day, and the 15 slowest rats were eliminated from the experiment.

The above five studies that did find evidence of a SucNCE also included controls for a SucPCE; none of these studies found evidence of a PCE. Thus, the conclusion that must be reached is that there is no substantial evidence for a SucPCE with shifts in delay; a SucNCE has appeared in only 5 of 13 published papers, and there is no understanding of the conditions that lead to its occurrence or nonoccurrence.

The evidence concerning simultaneous contrast effects is somewhat different. Five studies have reported clear evidence of a SimNCE (Beery, 1968; Chechile \& Fowler, 1973; Gavelek \& McHose, 1970; Mackintosh \& Lord, 1973; Sgro, Glotfelty, \& Podlesni, 1969), whereas 1 study (Logan, 1952) failed to find a SimNCE. The Logan study used a small difference in delays ( 1 vs. $5 \mathrm{sec}$ ) and a spatial discrimination choice apparatus that also involved a leverpress response. All of the other studies used runways-the Sgro et al. study used an Amsel double alley.

All of these studies, with the exception of Mackintosh and Lord, also examined for SimPCEs, and none found any evidence of it. Thus, SimNCE with differences in delay seems to be a robust phenomenon, whereas there is no evidence for SimPCEs.

\section{SHIFTS IN SCHEDULE OF REWARD}

Successive contrast effects apparently occur much more readily when the schedule is shifted than when delay is shifted. Leung and Jensen (1968) found evidence of SucPCEs when rats were shifted from $33 \%$ or $17 \%$ schedules to $100 \%$ reinforcement. SucPC was also obtained by Lehr (1974) in two experiments and by Shanab and Cavallaro (1975) in one experiment, all involving $50 \%$ to $100 \%$ shifts, by Seybert (1979) in a $33 \%$ to $100 \%$ shift, and by Fox (1972) and Dyck, Dresel, Thiessen, and DiLollo (1977) in $25 \%$ to $50 \%$ shifts. Only two studies failed to find SucPCEs: one by Roop and Nation (1976) in a 50\% to $100 \%$ shift and one by McCain, Lobb, and Newberry (1976) in a $25 \%$ to $75 \%$ shift. The Roop and Nation paper did report a SucPCE when punishment (shock) was administered concurrently with the nonreward prior to the shift to a $100 \%$ reward/ no-punishment schedule. SucPC has also been found when a period of extinction is interpolated between periods of $100 \%$ reinforcement (Harris, Smith, \& Weinstock, 1962), and Shanab, Birnbaum, and Cavallaro (1974) reported SucPCEs when animals were shifted from partial reward in a runway to continuous reward in an operant chamber. Finally, a SucPCE has been found when the shift was from $100 \%$ small reward to $50 \%$ large reward, $50 \%$ small reward (McHose \& Moore, 1978). Thus, the evidence for a SucPCE with schedule shifts is substantial.

The results are also clear in the case of negative contrast. All five papers that included the proper controls found evidence of a SucNCE when schedules were made leaner (Dyck et al., 1977; Fox, 1972; McCain et al., 1976; McHose \& Peters, 1975; Seybert, 1979). In the McCain et al. study, the NCE occurred only on the third shift in a LSLS sequence of shifts. In a varied-magnitude study, McHose and Moore (1978) found a SucNCE when rats were shifted from $100 \%$ large reward to $50 \%$ large reward, $50 \%$ small reward.

Fox (1972) reported that CEs could be obtained following shifts in N-length. That is, shifts in trial sequences in which the longest run of nonreward trials was 3 to a sequence in which the longest run was 1 were said to produce a PCE. The opposite shift was reported to lead to a NCE. However, Dyck et al. (1977) and Walkenbach, Haddad, and Mellgren (1978) have shown that these contrast effects were due to shifts in percentage, which were confounded with sequence in the Fox study. Shifts in N-length alone do not appear to produce contrast effects.

There is no substantial evidence of a SimPCE with variations in reward percentage (Galbraith, Rashotte, 
\& Amsel, 1968; Henderson, 1966; Spear \& Pavlik, 1966). McHose and Peters (1973) did report a SimNCE in running for 0 reward when the 0 reward was compared with 12 pellets on a $100 \%$ schedule vs. when the 0 reward was compared with 12 pellets on a $50 \%$ schedule. It is clear that there is no definitive evidence regarding the occurrence of SimCEs with variations in percentage reward.

\section{CONTRAST WITH AVERSIVE REINFORCERS}

There have been few studies in this category. Three of four studies investigating contrast in escape conditioning have found a SucNCE, and one of the four obtained a SucPCE. Bower, Fowler, and Trapold (1959) found neither CE with shifts in amount of voltage reduction in a runway escape task. However, Nation, Wrather, and Mellgren (1974) found both SucNCEs and SucPCEs in a similar study. The principal differences in the two studies seem to be that Nation et al. shifted current level and the animals were shocked only in the start and run segments of the alley. No shock was delivered in the goalbox. Bower et al. shifted voltage level and the animals were shocked throughout the runway. Woods (1967) found a SucNCE but no SucPCE in a water escape task with shifts in water temperature as the reinforcer.

McAllister, McAllister, Brooks, and Goldman (1972) took a different approach. They manipulated reward magnitude in escape conditioning by varying the similarity of the safe and shock chambers. McAllister et al. found a SucNCE in the third of three studies-the study in which there was the greatest difference between the two chambers. This latter finding parallels the general finding with appetitive reinforcers in that contrast is more likely to occur with larger reward shifts. The McAllister et al. study reported no evidence of a SucPCE in any of the three experiments.

Two studies have investigated reward shifts in a punishment paradigm. Nation, Mellgren, and Wrather (1975) found a SucNCE but no SucPCE in a shift of current levels used as punishment in a runway task. Shanab and White (1972) reported a SucPCE when an increase in reward from 1 to 12 pellets was combined with the removal of a shock punishment.

It is obvious that there have been far too few studies investigating SucCE with aversive reinforcers to permit any general conclusions. The McAllister et al. (1972) study does, however, indicate that it might be fruitful to manipulate variables that have been shown to be effective in determining degree of contrast in studies with appetitive reinforcers.

We found only one study that examined for SimCEs with an aversive reinforcer. Bintz (1971) varied cued shock intensity in a goldfish shuttle-avoidance task. There was some evidence of a SimPCE early in acquisition when a group which received both 12- and 6-V shocks performed better under the $12-\mathrm{V}$ condition than did fish that had always received $12-\mathrm{V}$ shocks. However, this result was made difficult to interpret by the fact that performance under the 6-V condition was also better than that of the constant $12-\mathrm{V}$ animals. Late in acquisition, performance under the 12-V condition, both between- and withinsubjects, was better than performance under the 6-V condition, with no evidence of SimPCE. SimNCE was indicated by the poorer performance under $12 \mathrm{~V}$ by fish that had experienced both 12 and $18 \mathrm{~V}$ as compared with the performance of fish under a constant $12-\mathrm{V}$ condition. However, this result was clouded by the fact that performance was also poorer under the $18-\mathrm{V}$ condition in the varied shock group than it was under the constant $12-\mathrm{V}$ condition. Thus, there was no clear evidence of either SimCE in this study.

\section{TRANSSITUATIONAL CONTRAST}

Premack (1969) addressed the question of the effect on contrast of the context in which a reward is received. Specifically, Premack wondered whether contrast would occur if animals received different levels of reward in different experimental situations. He attempted to answer this question by comparing the performance of rats repeatedly shifted from a situation in which running in a wheel was reinforced by the opportunity to drink milk on a mult VI VI schedule with performance in a situation in which barpressing on an FR schedule was reinforced with milk. In two of three rats tested, Premack found evidence that decreases in reinforcement frequency in the running wheel led to increased rates of responding on the FR schedule. In other words, two of the three rats showed a behavioral contrast effect that was transsituational.

In a runway study, Flaherty and Avdzej (1976) found that a negative contrast effect occurred in goal speed in rats running for a $4 \%$ sucrose reward when these same rats were also given $32 \%$ sucrose in a Plexiglas chamber in a different room. Contrast in consummatory behavior occurred in both situations.

There are apparently no other studies designed specifically to address this problem. However, there are some studies that might be considered related. For example, DiLollo (1964a) found that variations in runway-goalbox similarity did not affect the degree of SucNCE obtained, and E. D. Capaldi (1978) found that shifting alley brightness at the time of reward shift did not reduce a SucNCE. Shanab et al. (1974) found a SucPCE when animals were shifted from partial reinforcement in a runway to continu- 
ous reinforcement in an operant chamber compared with animals that were continuously reinforced in both (no partial reward controls were run).

McHose (1973) shifted reward either in the runway goalbox or outside the goalbox during the intertrial interval. He found that either shift led to a NCE in runway behavior. However, Calef et al. (1978) found that only changes in goalbox reward led to NCEs.

The little research that is available on this topic indicates that animals may compare rewards across quite different contexts (indeed, there is some data indicating that home cage feeding conditions may influence behavior in an experimental apparatusBacotti, 1976), but the topic needs systematic research.

\section{ONTOGENY OF CONTRAST}

Roberts (1966) reported that a SucNCE in runway behavior occurred in 180-day-old rats but not in 25 day-olds (actually 46-day-olds at the time of the shift). Systematic studies by Chen, Gross, and Amsel (1981) and Stanton and Amsel (1980) have provided substantial information on the ontogeny of SucNCEs in the runway. The first study found that 11-, 14-, and 16-day-old rats showed a drop in performance when reward (access to milk) was downshifted but did not show a NCE. The Chen et al. study found SucNCEs in 61-68-day-old rats and in 34-35-dayold rats, a marginal NCE in 25-26-day-old rats, and no NCE in 17-24-day-old rats. All of these data were collected with a food-pellet reward. With a milk reward, a NCE was obtained in 25-26-day-old rats, some evidence of it was found in rats 20-21 days old, but none was found in 16-17-day-old rats. Thus, a SucNCE may develop earlier with milk reward than with a food-pellet reward. An interesting aspect of this research was that partial reinforcement extinction effects were found in rats apparently too young to show NCEs-a result which indicates at least some separation of mechanism.

Fagen and Shoemaker (1979) investigated simultaneous contrast effects in the consumption of sucrose solutions. They found both SimPCEs and SimNCEs in rats aged $30,51,72$, and 93 days. This study was interesting in that it used both a longitudinal and a cross-sectional design. No substantial differences in the size of contrast as a function of age were found, although there was some indication that SimPCEs increased in size in the older rats when the cross-sectional design was used.

\section{CONTRAST FROM \\ A COMPARATIVE PERSPECTIVE}

There is substantial evidence that neither SucNCEs nor SucPCEs occur in goldfish or turtles following shifts in the magnitude of reward (Gonzalez, Ferry, \& Powers, 1974; Gonzalez, Holmes, \& Bitterman, 1967; Gonzalez, Potts, Pitcoff, \& Bitterman, 1972; Lowes \& Bitterman, 1967; Mackintosh, 1971; Pert \& Bitterman, 1970; Raymond, Aderman, \& Wolach, 1972; Wolach, Allen, \& Latta, 1974; Wolach, Raymond, \& Hurst, 1973).

Consistent with the failure to observe a SucNCE is the finding that turtles and goldfish given a large reward extinguish more slowly than animals given a small reward (Gonzalez et al., 1967, 1972; Mackintosh, 1971; Pert \& Bitterman, 1970). Bitterman (1975) has summarized the differences between goldfish, turtles, and rats in a variety of learning tasks.

The evidence is different in the case of SimCEs. SimNCEs have been readily obtained in goldfish, turtles, and pigeons (Brownlee \& Bitterman, 1968; Burns, Woodard, Henderson, \& Bitterman, 1974; Cochrane, Scobie, \& Fallon, 1973; Gonzalez \& Champlin, 1974; Gonzalez \& Powers, 1973; Pert \& Gonzalez, 1974). There is no evidence of a SimPCE in goldfish (e.g., Cochrane et al., 1973). Gonzalez and his colleagues (Gonzalez \& Champlin, 1974; Pert $\&$ Gonzalez, 1974) have made the point that SimNCEs and behavioral contrast seem to vary together and to be different from SucCEs.

One final study to be mentioned here is that of Bitterman (1976). Bitterman obtained evidence of a SucNCE in honey bees when the concentration of a sugar solution was shifted. This result seems paradoxical given the absence of SucCEs in fish and turtles. However, Bitterman's interpretation was that the CE that occurred in the bees was the result of sensory adaptation. When sensory adaptation was allowed to wane, no CEs occurred.

\section{CONTRAST IN CHOICE BEHAVIOR}

There have been several attempts to demonstrate contrast in choice subsequent to a shift in reward paired with one of two cues. The general procedure is exemplified by a study of Spear and Spitzner (1966). Rats were trained in a T-maze with different rewards (12 pellets or 1) correlated with different arms of the maze (the arms differed in brightness and texture). A second group of rats was always given 1 pellet in both arms. Experience with both alternatives was equated by the use of forced trials. After preshift training, the differential group (12 pellets vs. 1) was shifted to a 1-pellet reward in both arms. The results of several experiments of this general design (Spear \& Hill, 1965; Spear \& Spitzner, 1966, 1969b) indicated a SimNCE in running speeds during preshift, a weak SucNCE in speeds postshift, but no evidence of a contrast in choice behavior in the shifted rats. Contrast in choice was examined by comparing the choice of the shifted animals for 
the formerly large-reward arm with the choices of the 1-pellet control group. The choice of the differential group for the arm that once contained the 12pellet reward tended to remain above that of the 1-pellet group, rather than dropping below it as would have been the case had contrast occurred.

Singer (1973) also failed to find contrast in choice in a shift from sucrose solutions to a pellet, but a later study by Eisenberger, Frank, and Park (1975) found contrast in choice behavior in three experiments. Seemingly, an important procedural feature of the latter study was the elimination of preshift experience with simultaneous contrast and the postshift reward. That is, the animals in the Eisenberger et al. study received preshift experience only with the larger reward and then, postshift, were given a choice of going to the location in which a small reward had replaced the former large reward or of going to a different, novel location where the same small reward was found. The shifted animals tended to choose the familiar alley less often than a group that had always experienced small reward in the familiar alley. Eisenberger et al. also found positive contrast in choice behavior when reward was increased.

\section{CONTRAST WITH SUCROSE OR SACCHARIN SOLUTIONS}

This section will be concerned with contrast obtained with shifts in the concentration of sucrose or saccharin solutions. Several different paradigms will be examined: contrast in runway behavior with shifts in sucrose concentration; contrast in consummatory behavior with relatively brief exposure to the solutions; and, finally, contrast in saccharin consumption with extended exposure (the "saccharin elation effect").

\section{Shifts in Sucrose Concentration and Runway Contrast}

The major part of the data to be considered in this section can be summarized briefly: successive negative contrast seems not to occur in runway behavior when the concentration of sucrose solutions is shifted downward. An impressive number of studies have obtained this result with wide variations in parameters (Barnes \& Tombaugh, 1973; Burns \& Burns, 1978; Burns, Dupree, \& Lorig, 1978; Collier, Knarr, \& Marx, 1961; Flaherty \& Caprio, 1976; Flaherty, Riley, \& Spear, 1973; Goodrich \& Zaretsky, 1962; Homzie \& Ross, 1962; Rosen, 1966; Rosen \& Ison, 1965; Spear, 1965; Shanab, France, \& Young, 1975).

This failure of SucNCE to occur in runway behavior has also been found when the concentration of sucrose pellets is shifted down (Shanab, France, \& Young, 1975). However, contrast does occur when the quantity of sucrose pellets or of liquid sucrose is shifted down (Shanab et al., 1975, 1976; Burns et al., 1978). At one time there was evidence that durable SucPCEs and SucNCEs occurred in leverpress behavior when sucrose concentration was varied (Collier \& Marx, 1959). However, Dunham and Kilps (1969) have shown that this was due to variations in the body weight of the different groups. When body weight was equated, no contrast occurred.

The failure of contrast to occur in instrumental behavior has been found in experiments in which a shift in liquid sucrose has produced a large negative contrast in consummatory behavior in the goalbox (Flaherty \& Caprio, 1976). In fact, there is some evidence that there is little or no relationship between consummatory behavior for sucrose solutions and measures of runway behavior preceding the consummatory behavior (Flaherty \& Caprio, 1976; Knarr \& Collier, 1962).

The failure of contrast is not understood. At one time, it seemed plausible that different concentrations of sucrose solutions did not leave distinct "aftereffects," thereby making the discrimination of the postshift solution from the preshift solution difficult (e.g., Likely, Little, \& Mackintosh, 1971). However, experiments have shown that contrast readily occurs in consummatory behavior when sucrose solutions are shifted (see below), even with retention intervals of 10 days or longer inserted between the last experience with the preshift reward and the first experience with the postshift reward (e.g., Ciszewski \& Flaherty, 1977; Flaherty \& Lombardi, 1977) and that patterning behavior occurs in the runway with different concentrations of sucrose, thereby implying that discriminative aftereffects of different sucrose solutions may enter into instrumental associations (Burns, 1976).

Another possibility is that shifts in sucrose concentration, unlike shifts in food, are not aversive and that aversiveness is necessary for a contrast effect to occur. However, Daly (1974a) has shown that rats shifted to extinction after receiving $20 \%$ sucrose reward in a runway will learn a hurdle jump response in order to escape from extinction-related cues. This behavior is the same as that shown in animals shifted to extinction after being rewarded with food pellets (Daly, 1974a). Thus, by this measure of aversiveness, there seems to be no difference between shifts in sucrose and solid food, when the shift is to zero reward.

The results obtained with successive sucrose shifts in the runway are made more puzzling by the fact that SucNC apparently does occur in operant situations with a downshift in sucrose concentration (Weinstein, 1970a, 1970b) and that SimNCEs do occur in the runway with variations in the same sucrose concentrations that fail to produce SucNC (Flaherty et al., 1973). 


\section{Shifts in Sucrose Concentration}

\section{and Consummatory Contrast}

If rats are allowed access to a tube containing $32 \%$ sucrose for a 5-min period each day, and then, after a number of days, the concentration is shifted to $4 \%$, a SucNCE will occur in number of licks made to the postshift solution (e.g., Flaherty, Capobianco, \& Hamilton, 1973; Flaherty \& Hamilton, 1971; Vogel, Mikulka, \& Spear, 1968). These contrast effects are highly reliable, with the lick rate of shifted animals often $50 \%$ or less of the lick rate of unshifted controls. They have been obtained when the sucrose is presented in small chambers, when it is the reward for runway behavior (Flaherty \& Caprio, 1976), and when presented in an open-field apparatus (Flaherty, Blitzer, \& Collier, 1978). These SucNCEs have also been obtained in both free-feeding and deprived rats (Riley \& Dunlap, 1979).

Contrast effects of this type are uninfluenced by lesions of the septum (Flaherty, Capobianco, \& Hamilton, 1973; Flaherty \& Hamilton, 1971; Flaherty, Powell, \& Hamilton, 1979). The effect of hippocampal lesions is not clear. Murphy and Brown (1970) found small, but nonsignificant, negative contrast effects in lesioned rats following a $32 \%$ to $4 \%$ sucrose shift. However, Kramarcy, Mikulka, and Freeman (1973) found unchanged negative contrast effects following a similar shift in rats with dorsal hippocampal lesions. Whether this difference in results is related to lesion location or to many other procedural differences remains to be determined. Earlier in this paper, we mentioned that hippocampal lesions apparently eliminate negative contrast in runway behavior following a shift in number of pellets (Franchina \& Brown, 1971).

A number of drug studies have indicated the following. Chlordiazepoxide, when given during both preshift and postshift periods, does not influence SucNC (Flaherty, Lombardi, Wrightson, \& Deptula, 1980). However, when chlordiazepoxide is injected for the first time on the 2nd or 3rd postshift day, contrast is greatly reduced or eliminated. Injecting on the 1st postshift day has little effect on contrast (Flaherty et al., 1980; Vogel \& Principi, 1971).

Amobarbital sodium has somewhat different effects. Like chlordiazepoxide, amobarbital sodium has no effect on SucNC in consummatory behavior when injected preshift and postshift. However, injections of this drug for the first time on the 1st postshift day reduced contrast, a result also found when the drug was injected for the first time on the 2nd postshift day (Flaherty, Becker, \& Driscoll, 1982; Flaherty \& Driscoll, 1980). The effects of both chlordiazepoxide and amobarbital sodium on postshift consummatory behavior might reflect a disinhibitory effect of the drug, resembling the effects of a novel tone introduced during the postshift period (Lombardi \& Flaherty, 1978). These results, obtained with chlordiazepoxide and amobarbital sodium on consummatory contrast, differ from those obtained with the same drugs on runway contrast with shifts in solid food rewards. In the latter situations, the application of either drug during the preshift and postshift periods eliminates SucNCE (Rosen, Glass, \& Ison, 1967; Rosen \& Tessel, 1970). Comparable drug doses were used in the relevant experiments.

Another drug that has been investigated is scopolamine, a compound that has apparent inhibitionreducing properties in some test situations (e.g., Bohdanecky \& Jarvik, 1967; Carlton, 1969). However, scopolamine does not reduce the degree of SucNCE in consummatory behavior, either when applied preshift and postshift or when applied postshift only. In fact, scopolamine tends to reduce the consumption of the $4 \%$ sucrose solution, an effect apparently related to the centrally acting, rather than peripherally acting, properties of the drug (Flaherty \& Meinrath, 1979).

Finally, the effects of ip injections of ethanol on contrast have been investigated. Like chlordiazepoxide and amobarbital sodium, ethanol seems to be more effective in reducing SucNC in consummatory behavior when the first injection of the drug is made on the 2nd postshift day; injections on the 1st postshift day are without effect (Becker \& Flaherty, 1982). A dose-response function was obtained in this study for the effects of ethanol on the 2nd postshift day. A .75- and a $1-\mathrm{g} / \mathrm{kg}$ dose of a $15 \%$ ethanol solution had contrast-reducing effects. Larger doses $(1.5$ and $2.0 \mathrm{~g} / \mathrm{kg})$ debilitated the animals, and smaller doses $(.25$ and $.50 \mathrm{~g} / \mathrm{kg}$ ) were ineffective in reducing contrast.

Successive negative contrast in consummatory behavior shows an orderly rate of loss over retention intervals inserted between preshift and postshift periods. Levels investigated have included $1,4,5$, 10,17 , and 32 days. Contrast has been found to be unreliable at 32 days postshift and marginally reliable at 17 days postshift (Ciszewski \& Flaherty, 1977; Flaherty, Capobianco, \& Hamilton, 1973; Flaherty \& Lombardi, 1977; Gordon, Flaherty, \& Riley, 1973). Reinstatement treatments, consisting of brief access to $32 \%$ sucrose, administered during a 17-day retention interval do not seem to prolong memory (Ciszewski \& Flaherty, 1977). Contrast may also be obtained with as little as $5 \mathrm{~min}$ exposure to the $32 \%$ sucrose prior to the shift to $4 \%$. These contrast effects have been found to diminish with increasing retention intervals from $20 \mathrm{~min}$ to $70 \mathrm{~h}$ after the initial exposure, but they were still statistically reliable after the longer interval (Flaherty, Ciszewski, \& Kaplan, 1979).

Downshifting the concentration of sucrose available in an open-field environment leads to increases in ambulatory behavior and rearing, in addition to a 
negative contrast in lick rate. Grooming and stationary behaviors are not much affected by such shifts, and there is no evidence of an increase in defecation as a result of the shift. These open-field studies have also indicated that rats of both sexes and rats derived from Long-Evans, Sprague-Dawley, and Wistar strains all behave in a qualitatively similar manner following the sucrose shifts (Flaherty et al., 1978, 1979; Flaherty, Troncoso, \& Deschu, 1979).

There has been some evidence of a SucPCE obtained in sucrose consumption (Lombardi, 1978; Panksepp \& Trowill, 1971; Premack \& Hillix, 1962) but, as in the case of other positive contrast paradigms, little in the way of parametric or analytic studies.

\section{Simultaneous Contrast}

The consummatory contrast paradigm has also yielded substantial evidence of SimPCE and SimNCE effects. The procedure used in these experiments involves alternating brief access periods to two tubes containing sucrose solutions and the comparison of lick rates for a particular concentration when the alternative tube has a different concentration and when the alternative tube contains the same concentration. These experiments have shown that SimPCEs and simNCEs occur in measures of lick rate, intake, and latency to switch tubes; occur with access periods ranging between 15 and $60 \mathrm{sec} /$ tube; tend to increase from the first to the third access period to each tube; occur with between- as well as within-subjects controls; are not reduced by administration of chlordiazepoxide, imipramine, scopolamine, or amobarbital sodium; and are not subject to disinhibition by the presentation of a novel stimulus (Flaherty \& Avdzej, 1974; Flaherty, Becker, \& Driscoll, 1982; Flaherty \& Largen, 1975; Flaherty \& Lombardi, 1977; Flaherty, Lombardi, Kapust, \& D'Amato, 1977; Flaherty \& Meinrath, 1979; Flaherty, Wrightson, Deptula, \& Duston, 1979; Lombardi \& Flaherty, 1978).

When the concentrations of sucrose available in the two tubes is varied systematically (i.e., $32 \%$ vs. $2 \%, 32 \%$ vs. $4 \%, 32 \%$ vs. $8 \%$, and $32 \%$ vs. $16 \%$; or $16 \%$ vs. $2 \%, 4 \%, 8 \%$, and $12 \%$ ) within-subjects, the lick rates of the animals also varies in a systematic fashion. In particular, the proportion of licks made to the tube containing the higher concentration tends to match the "proportion of concentration" available from that tube. These data were also examined in terms of ratios rather than proportions. An exponential function was obtained when the ratio of licks made to the higher of the two concentrations was plotted against the ratio of the concentration values. The exponent of this power function was found to be 1.15 and 1.13 in the two series of sucrose pairs listed above (Flaherty \& Kaplan, 1979; Flaherty \& Sepanak, 1978).
The results of all these experiments on SimCEs with sucrose have been interpreted as indicating that such contrast effects are controlled simply by taste factors, perhaps much like those governing human judgments of relative sweetness.

\section{Saccharin Elation Effect}

Another apparent contrast phenomenon that is probably also related to taste is the "saccharin elation effect." This effect occurs when animals are given daily access to saccharin, usually in the home cage, for an extended period and then the saccharin is removed for several days and, finally, returned again. Saccharin intake on the 1st day of the return is usually substantially greater than in the baseline period, before removal. Typically, animals are maintained on ad-lib food and water throughout the course of these experiments.

These elation effects have been demonstrated with access periods to saccharin ranging from $10 \mathrm{~min}$ per day, to $1 \mathrm{~h}$ per day, to continuous access (Ashton, Gandelman, \& Trowill, 1970a; Ashton \& Trowill, 1970; Dube, Ashton, \& Trowill, 1970; Gandelman \& Trowill, 1969; Hoyenga \& Hoyenga, 1973; Pinel \& Rovner, 1977). However, there have also been failures to demonstrate the effect (DiLollo \& Meyer, 1970), and it has been argued that the elation effect is more reliable when animals are given continuous access to saccharin before the period of absence (Pinel \& Rovner, 1977). This parameter of duration of daily exposure may be important for the integration of the saccharin elation effect with other possible taste-contrast phenomena. In particular, an elation pattern has failed to occur in two studies in which sucrose solutions were used in the standard saccharin elation paradigm (Ashton, Gandelman, \& Trowill, 1970b; Ashton \& Trowill, 1970), and yet, an effect similar to the saccharin elation effect has been repeatedly demonstrated with ethanol and quinine solutions (e.g., Pinel \& Huang, 1976; Sinclair, Walker, \& Jordan, 1973; Wayner, Greenberg, Tartaglione, Nolley, Fraley, \& Cott, 1972). It is possible that duration of exposure is responsible for the atypical results obtained with sucrose, since briefer exposures $(10 \mathrm{~min}$ or $1 \mathrm{~h})$ were used in these studies than were typical of the other studies (continuous).

Pinel and Huang (1976) have suggested that the saccharin elation effect, the alcohol deprivation effect, and possible similar effects found with other sapid solutions might be related to an inhibitory process that develops with repeated exposure to a given taste and then dissipates during the period of $a b$ sence. As yet, there has been no independent evidence demonstrating the existence of such an inhibitory process, but the data do indicate that the manner of exposure to a sapid solution (continuous or intermittent) may have a potent effect in determining the amount consumed. There has been no work con- 
ducted to determine the relationship of these "elation" effects to the contrast effects that occur with concentration shifts discussed in the previous sections. However, it seems possible that these elation effects may be related to the SucPCEs that occur in instrumental behavior when a period of nonreinforcement is interpolated between periods of reinforcement (Harris et al., 1962; Shanab et al., 1974; Vogel, Mikulka, \& Spear, 1966).

\section{Anticipatory Contrast}

Recent studies (Flaherty \& Checke, 1982) have shown that contrast may occur prior to the occurrence of a preferred substance. The frequency at which rats licked from either a $.15 \%$ saccharin or a $4 \%$ sucrose solution was lower when these solutions were followed $5 \mathrm{~min}$ later by $32 \%$ sucrose than when no second substance was presented. In addition, it was shown that the intake of $.15 \%$ saccharin was not reduced when the second substance was $2 \%$ sucrose, a solution whose hedonic value approximates that of $.15 \%$ saccharin. Thus, there is a reduced intake of the first substance only when there is a preference disparity. In these experiments, the solution pairs were presented once per day.

Evidence that these contrast effects were anticipatory in nature came from the observation that the degree of suppressed intake of the first substance was inversely related to the interval between the two solutions over the range investigated ( $15 \mathrm{sec}$ to $30 \mathrm{~min}$ ). If the contrast evident in the intake of the first substance presented daily had been due to a comparison of the saccharin or $4 \%$ sucrose with the memory of the $32 \%$ sucrose received on the previous day, then the relatively limited intersolution interval should have had little effect. The fact that these brief intervals were influential in controlling intake thus indicated that the comparisons between substances were in a forward direction.

\section{EXPLANATORY CONSTRUCTS}

A variety of theoretical constructs have been used to "explain" incentive contrast effects. We shall consider how several of these constructs have been applied as explanations of contrast and how these explanations fare in the light of the data reviewed above. The constructs to be considered are the following: generalization decrement, neophobia, inhibition, adaptation level and related concepts (incentive averaging and reinforcement level), frustration and related motivational-emotional concepts, exploration, and, finally, sensory processes.

\section{Generalization Becrement}

Perhaps the most theoretically conservative position is an interpretation of contrast in terms of generalization decrement. This position was developed largely to explain successive negative contrast effects by Spear (Spear \& Spitzner, 1966) and by E. J. Capaldi (e.g., E. J. Capaldi \& Lynch, 1967). According to this approach, the successive negative contrast effect may be attributed entirely to generalization decrement. The reasoning here is that the reward is one stimulus among many in the context of which the instrumental response is learned. When reinforcement conditions are changed, the stimulus situation is different from that prevailing during initial learning, and, thus, a decrement in performance ensues. When this new stimulus becomes associated with the instrumental response, performance returns to the level appropriate for that reward. This approach, which has been formally developed by E. J. Capaldi (1972) and by E. J. Capaldi and Lynch (1967), has advantages in that it is relatively parsimonious and employs a concept that has empirical support (e.g., E. D. Capaldi, 1978; Huang, 1969).

However, this approach also has disadvantages. Among these is the fact that this theory can be directly applied only to successive negative contrast effects. It cannot be used without modification to explain simultaneous negative contrast effects because the animals in a simultaneous contrast paradigm are repeatedly undergoing shifts in reward magnitude, but the contrast effect does not go away as a generalization decrement interpretation would predict; rather, the contrast effects in this paradigm seem to be permanent (e.g., Bower, 1961; Flaherty et al., 1973, 1977). In addition, the effects of deprivation state on contrast are not entirely consistent with a generalization-decrement interpretation. Successive negative contrast tends to vary directly with degree of deprivation, but a generalization-decrement interpretation would predict an inverse relationship, since generalization itself is often found to be greater with increasing amounts of deprivation (e.g., Kalish \& Haber, 1965). The evidence here, however, is not entirely clear (cf. Thomas \& King, 1959).

Another difficulty for generalization decrement as a satisfactory explanation of negative contrast is presented by data obtained in repeated shift studies. E. J. Capaldi and Lynch (1967) reported that contrast occurred only with the first downshift in reward in a runway study. The failure to obtain a SucNCE with a second shift was taken as support for the generalization-decrement theory on the assumption that the postshift experience served to accrue habit strength to the new reward and, thus, there was no generalization decrement (and no contrast) on the second shift. Furthermore, E. J. Capaldi (1972) found that contrast was reduced by prior experience with the small reward if trials were massed, and that contrast was eliminated by prior experience with the small reward if trials were spaced. E. J. Capaldi attributed these differences between massed and spaced trials to two sources of generalization decre- 
ment-one short-lived and related to emotional events and one long-lived and related to memory of characteristics of the reward.

However, there are several studies that have shown that contrast occurs after the animals have had prior experience with the postshift reward, and even after they have experienced more than one shift (Calef et al., 1978; Maxwell et al., 1976; McCain \& Cooney, 1975; Shanab et al., 1978; Shanab \& Spencer, 1978). Furthermore, in several repeated-shift studies measuring consummatory behavior for sucrose, it has been shown that SucNCEs occur readily on the second and third shifts. Moreover, if animals are shifted every day (32-4-32-4, etc.), negative contrast in consummatory behavior has remained undiminished after nine shifts to the lower concentration. Repeated positive contrast effects were found only in the first three shifts, but the loss of positive contrast seemed to be due to the control group's reaching the same asymptote in lick rate that the shifted group had reached earlier. Thus, the lack of SucPCEs with the later shifts may have been a ceiling effects problem. Similar results were obtained when animals were repeatedly shifted after two experiences with each solution (Flaherty, Becker, Checke, \& Kalucki, Note 2). Thus, the effect of prior small-reward experience offers, at best, only partial support for a generalization-decrement interpretation of negative contrast.

Another problem is presented by a study in which a novel stimulus is introduced coincidentally with the postshift reward. When this was done in a sucrose consummatory experiment, contrast was reduced (Lombardi \& Flaherty, 1978) rather than being enhanced, as might have been expected from the generalization-decrement viewpoint-on the assumption that the novel stimulus and novel reward should have cumulative decremental effects. It should be noted that a decrement was found under these conditions in a simultaneous contrast study, but not in a successive contrast procedure (Lombardi \& Flaherty, 1978). In another study, in which runways were shifted coincidentally with a reward shift, a larger drop in running speed was found in the runway-shifted animals, but this effect was not reliable (E. D. Capaldi, 1978).

Thus, while the concept of generalization decrement would seem to apply logically to SucNCEs, it is not satisfactory as a complete account of the phenomenon. It also cannot function as an explanation of positive contrast, since generalization decrement can only interfere with the occurrence of positive contrast. When the original versions of the generalization-decrement interpretation were offered, there was relatively little evidence that successive positive contrast was a viable phenomenon. However, the present review shows that there have been many subsequent studies demonstrating the viability of positive contrast.

\section{Neophobia}

A concept related to generalization decrement, the concept of neophobia, has also been offered as an explanation for at least some aspects of SucNC. Generalization decrement and neophobia, as constructs, seem to differ in the following way. Generalization decrement refers to deterioration in a learned response which results from a shift to a stimulus context different from the one in which the response was learned. Neophobia is applied to situations in which an animal is shifted to a novel food substance or to a novel environment (Barnett, 1963; Cowan \& Barnett, 1975; Mitchell, Fairbanks, \& Laycock, 1977). Neophobia carries less of a connotation of a learned response than does generalization decrement, although there must obviously be some kind of learning; there can be no "neo" without a familiar.

The neophobia or hesitancy with which rats consume novel foods has been applied as an explanation of the SucNCEs that occur in consummatory behavior when sucrose solutions are shifted (Flaherty et al., 1980). In particular, it has been argued the neophobia may be the principal cause of such contrast on the 1st postshift day, whereas other processes may be involved on subsequent days. The reason for thinking that different processes may be involved on different postshift days is the evidence that several drugs (chlordiazepoxide, ethanol, sodium amobarbital) are more effective on Postshift Day 2 than on the Postshift Day 1. Indeed, chlordiazepoxide and ethanol seem to be without effect on Postshift Day 1 (see evidence reviewed above). In addition, it was found that a novel stimulus has a greater disinhibitory effect subsequent to the 1st postshift day (Lombardi \& Flaherty, 1978).

Other evidence in favor of this explanation has been circumstantial or difficult to explain. For example, Lombardi $(1978,1980)$ obtained results indicating that the experience of a downshift in sucrose concentration increased the rats' tendency to avoid novel flavors paired with a $12 \%$ sucrose solution. This generalized avoidance occurred both when a particular flavor was paired with the shift and when no flavor was paired with the shift. Lombardi (1978) also found some evidence that the experience of a contrast effect in consummatory behavior enhanced the conditioned aversion shown when $\mathrm{LiCl}$ was paired with a particular flavor.

Recent experiments that have attempted to directly manipulate susceptibility to neophobia have provided equivocal evidence. Meinrath (1980) attempted to manipulate neophobia in several different ways. Exposing rats to the Denenberg-Levine early- 
handling procedure (e.g., Denenberg \& Grota, 1964) was found to increase ambulation and decrease defecation in the open field and to produce a slightly faster recovery from contrast, but there was no effect on degree of contrast that occurred on the 1st postshift day, when neophobia should be most likely to operate. (Daly and Rosenberg, 1973, found no effects of early handling on SucNCE in a runway study.)

Similarly, exposing animals to varied taste experience just after weaning did not influence contrast experienced when the animals were adults. However, if varied tastes were administered to the animals in the preshift sucrose solutions, then degree of contrast was reduced on the 1st postshift day, but there was no effect on this treatment on recovery from contrast. Thus, the Meinrath experiment provided some evidence for two processes involved in SucNCEs with sucrose in that the varied taste experience influenced the 1st postshift day, but not recovery, whereas the early handling influenced recovery, but not the 1st postshift day.

\section{Inhibition}

Black (1968) proposed a model utilizing an inhibitory construct to explain SucNCEs. He assumed that behavior in a runway situation was related to "effective excitatory potential" $(\overline{\mathrm{E}})$, where $\overline{\mathrm{E}}=\mathrm{E}-\mathrm{I}, \mathrm{E}$ being excitatory potential, or, behaviorally, the tendency to run, and I representing inhibition which acts to reduce $\overline{\mathrm{E}}$. Black further assumed that $\mathrm{E}$ was incremented whenever a response was reinforced and that $I$ is incremented whenever a reinforcement smaller than what was "expected" occurred. Black also assumed that $E$ itself was an absolute function of amount of reward, that is, was not subject to contrast or relativity effects. The application of this equation is similar to the application of the generalization-decrement concept. For example, the equation applied to the Crespi paradigm predicts a SucNCE because the downshifted group should be inferior to the control group. Like generalization decrement, this equation does not predict or describe SucPCEs. However, unlike generalization decrement, this equation does seem to apply to the differential conditioning task. In this case, $\bar{E}=E$ for $S+$ but $\bar{E}=E-I$ in the $S-$ alley. Inhibition occurs in S- because the reward obtained there is less than the average of the rewards obtained in the situation. Thus, this theory predicts a SimNCE but not a SimPCE. In fact, if generalization of $I$ to $S+$ is considered, then the theory predicts the opposite of positive contrast, that is, lower performance by the differential group in $\mathrm{S}+$ than by a large-reward control group. This latter result has been obtained in some experiments (e.g., Bower, 1961; Flaherty et al., 1973; Matsumoto, 1969; McHewitt, 1974; McHose \& Howard, 1973). However, SimPCEs are also sometimes obtained (see above), and this model clearly does not account for them.

Certain aspects of models proposed by McHose (1970) and by Capaldi (Note 3) also employ an inhibitory construct. These models will be considered in another context below. There has been very little research specifically addressed to the involvement of inhibition in contrast. In a series of three experiments, Lombardi and Flaherty (1978) found that the presentation of a novel stimulus during the postshift phase of a SucNCE experiment diminished degree of contrast on the 2nd and subsequent postshift days. No effect was found on the 1st postshift day. Controls included in these experiments obviated explanations in terms of the energizing effects of the tone or the rate-dependent energizing effects of the tone. The interpretation offered was that these results represented a disinhibiting effect of the tone, thus supporting the use of inhibitory constructs to explain SucNCEs. No disinhibitory effect of a novel tone was obtained in three additional experiments using a SimNCE procedure. All of these experiments involved consummatory response measures and not instrumental behavior.

A subsequent search for evidence of conditioned inhibition as a result of pairing stimuli with a SucNCE was, however, without success (Lombardi, 1980). Instead of conditioned inhibition, the evidence indicated that a generalized neophobic response occurred as a result of negative contrast experience. Thus, direct investigation of the role of inhibition in negative contrast has led to some, but not complete, support for the ideas embedded in the Black and McHose models. Also, as stated above, neither of these models is applicable to PCEs.

\section{Adaptation Level, Incentive Averaging, and Reinforcement Level}

The adaptation level theory of Helson (1964) has been applied to reward contrast by Bevan in a series of articles $(1963,1966,1968)$. In psychophysical or scaling research, it has been shown that judgments concerning a given point along a stimulus dimension, such as weight of lifted objects, are not determined solely by the value of the given point. Such judgments are also influenced by the context in which they are made (e.g., DiLollo, 1964b). The adaptationlevel interpretation of results such as this is that subjects pool all relevant stimuli to form a norm or adaptation level which is used as a standard in judgments concerning individual points along the stimulus dimension. An interpretation of the contrasteffect paradigm in terms of this theory is the following: Subjects judge reward magnitudes by comparing each particular value of reward with an adaptation level formed on the basis of past or concurrent experience with other rewards. Thus, subjects in a downshifted group "judge" the small reward to be 
smaller than do control subjects that have not had a prior history of larger reward. Similarly, in the case of upshifts in reward magnitude, the new reward apparently "appears" larger to the shifted group than to the unshifted control group. Thus, the adaptation-level theory predicts both positive and negative contrast effects, a factor that has been considered a weakness in the theory in the past (e.g., Black, 1968) but now may be considered an asset in light of the recent data indicating the viability of SucPCEs.

The application of adaptation-level theory to contrast effects has, however, been criticized (Spear, Note 4). In his analysis of the distribution of running times obtained in a differential conditioning task, Spear found that the time scores of the small-reward control group and the time scores of the differential group to $S+$ were normally distributed, but the time scores of the differential group to $S$ - were skewed in the direction of having some very long latencies. This skewed distribution indicated that something other than the misjudgment of stimulus attributes was involved in the SimNCE. Spear reasoned that differences in judgments of stimulus attributes would be likely to produce a difference in the central tendency of the small-reward control group and the differential group but not a difference in skewness. Instead, it seems that the skewness of the $S$ - distribution is more likely produced by the running behavior's being disrupted by emotional responses rather than by bad judgments.

The incentive-averaging concept of McHose (1970; McHose \& Moore, 1976; McHose \& Peters, 1975) and the reinforcement-level concept of E. J. Capaldi (1974) are similar in principle to the adaptationlevel concept, but they do not carry the connotation of a perceptual judgment. McHose assumes that the degree of negative contrast is proportional to the degree of decrement between the postshift incentive and the average value of the preshift incentive. The average preshift incentive value is a function of the magnitude and frequency of the different incentives received during the preshift period. These assumptions are consistent with the facts that degree of NCE does vary with degree of reward disparity and with the average preshift reward obtained in partialreward and varied-magnitude-reward schedules (see above). The incentive-averaging concept also seems to be applicable when animals are shifted to a partialreward schedule in the postshift period (McHose \& Peters, 1975) and when partial reward precedes an upshift in reward (Lehr, 1974). Incentive averaging is a valuable descriptive concept and one that may also have some power of making quantitative predictions (McHose \& Peters, 1975).

E. J. Capaldi's reinforcement-level theory is consistent with the incentive-averaging approach in that it assumes that expected reward increases as magnitude and percentage of reward increase, and that contrast is due to the difference between expected reward and obtained reward. It is this relationship that is specified as reinforcement level. Capaldi's model is more ambitious than the incentive-averaging model in that it specifies a process by which contrast occurs and the process is assumed to be associative. That is, the strength of S-R associations is determined by reinforcement level. If obtained reward is greater than expected reward, stimuli will acquire a greater propensity to elicit a response than if obtained reward equals expected reward (E. J. Capaldi, 1974, p. 958). Conversely, if obtained reward is less than expected reward, the propensity of stimuli to elicit a response will decrease (termed "unconditioning').

This and a more formal version of the model (Capaldi, Note 3) make some specific predictions. For example, SucPCEs should be long lasting. This sometimes happens (e.g., Mellgren, 1971a, 1973; Seybert, 1979), but sometimes does not (e.g., McHose \& Moore, 1978; Premack \& Hillix, 1962; Seybert \& Mellgren, 1971). McHose and Moore, whose paper was designed to compare the magnitude and the duration of both contrasts, found them similar in magnitude and SucPCEs to be of relatively short duration. The discrepancy in duration of PCEs is not accounted for by the model or by a review of the literature.

The model predicts that SucNCEs should be greater with increasing numbers of preshift trials. This seems to be the case (e.g., Vogel, Mikulka, \& Spear, 1966).

The model predicts that SucNCEs should be less if the animals have had prior experience with the small reward. As reviewed above, the data on this topic are mixed, but there is no strong support for this conclusion.

The model predicts that contrast should occur with shifts in N-length. As reviewed above, Fox (1972) appeared to find this result, but Dyck et al. (1977) and Walkenbach et al. (1978) were able to show that these results occurred because Fox confounded percentage with $\mathrm{N}$-length. When $\mathrm{N}$-length alone was shifted, no contrast was found.

Predictions made by the model regarding the extinction effects produced by upward reward shifts superimposed on partial reinforcement schedules have not been supported (Nation \& Durst, 1980).

As mentioned above, the model is ambitious in attempting to account for a variety of contrast and partial reinforcement phenomena (Capaldi, Note 3) and in attempting to provide a comprehensive theory of the process behind contrast. However, the theory seems, at least to the present reviewer, to be inconsistent with recent theoretical developments in learning. For example, it is surprising that $E$. J. Capaldi still uses a Hullian S-R habit concept as it was used in the 1940s. There is no mention of intervening research which has indicated that S-R connections may not be the best way to conceptualize instru- 
mental learning (e.g., Hearst \& Peterson, 1973), no mention of the importance of contingency in learning (e.g., Rescorla, 1968, 1969)-Capaldi assumes that expectancies are learned by contiguity-and no mention of recent theoretical developments in learning that have emphasized Tolmanian constructs (e.g., Bolles, 1972; Bindra, 1972, 1974). Capaldi uses a Hullian reinforcement principle for habit strength despite the fact that it is outmoded. To quote Pert and Gonzalez, "contemporary theorists are almost unanimous in their conviction that reward does not play an associative role in instrumental learning" (Pert \& Gonzalez, 1974, p. 536).

It also seems unusual that E. J. Capaldi made reward expectancy-a Pavlovian process in his theory - to be resistant to change, whereas habit strength -an instrumental process-was made more susceptible to rapid change. This viewpoint seems contrary to the very developments that Crespi's original findings stimulated (see above and Spence, 1956). Of course, none of this is necessarily bad if the model has heuristic value.

In summary, some concept like adaptation level would appear to be an essential component of the eventual explanation of contrast. It is clear that contrast is related to the discrepancy between preshift reward events and postshift reward events and that both kinds of events must be broadly defined (i.e., must take into account magnitude, percentage, and frequency of different reward instances in both preand postshift phases). It is also possible that overall and momentary incentive averaging may be important in determining degree of contrast. That is, the value of recent reward events may weigh more heavily than remote reward events in determining average incentive value. Finally, either of these two incentive averages may be influenced by the particular sequences in which reward events are received (see Reward Sequence Effects above).

\section{Frustration and Related \\ Motivational-Emotional Processes}

Attempting to interpret contrast in terms of emotional responses leads to all of the problems associated with the concept of emotion in animals. This reviewer is unaware of any studies that have incorporated psychophysiological measures, such as skin resistance, into a contrast experiment. However, evidence in favor of the involvement of emotional responses in contrast may be derived from a number of indirect sources.

The first source is a combination of behavioral observation and anthropomorphic inference. The quote from Tinklepaugh on the first page of this review exemplifies the use of an emotionally descriptive adjective to describe the consequences of a downshift in reward. Similarly, Crespi's choice of the terms "elation effect" and "depression effect" were clearly influenced by his preference to interpret the "overshooting" and undershooting" obtained in his study as emotionally based phenomena, perhaps analogous to the "abortive sorrows and short lived elations of men" (Fitzgerald, 1953). Crespi (1944) wrote that shifts in reward magnitude produced a heightened emotional drive-elation or joy, which enhanced ongoing behavior in the case of upshifts in reward, and anger or frustration, which subtracted from ongoing behavior in the case of downshifts in reward. The mechanisms by which these emotional responses came to affect behavior were not clearly specified. Crespi did, however, describe some of the behavior that was associated with the slower running of the downshifted animals. This behavior included refusals to eat the 16-unit reward, "attempts to jump out of the goal box," attempts at retracing, "peering into the food box," and hesitant running. As post-Crespi research through the 1960 s began to indicate that positive contrast effects were, at best, an elusive phenomenon, theoretical efforts focused almost exclusively on negative contrast effects. Bower (1961) suggested that SimNCEs might be explainable in terms of Amsel's (1962) frustration theory. Bower reasoned that the lowered performance in S- by the differential group, relative to the small-reward control group, might be the result of conflict between the anticipation of reward $\left(r_{g}\right)$ and the anticipation of frustration $\left(r_{f}\right)$, and that frustration occurred because of generalization of large reward $r_{\mathrm{g}}$ from $\mathrm{S}+$ to $\mathrm{S}-$. This interpretation of SimNCEs was elaborated by Ludvigson and Gay (1967). These investigators recognized two possible mechanisms for the occurrence of $r_{f}$ in the $S-$ alley. One was, as Bower suggested, the elicitation of large reward $r_{g}$ in the $S-$ alley by a process of stimulus generalization. A second possible mechanism was the elicitation of an intermediate value of $\mathrm{r}_{\mathrm{g}}$ in $\mathrm{S}-$ by situational cues common to both alleys. This intermediate $r_{g}$ would still be larger than the small reward, and thus frustration would still occur. The Ludvigson and Gay (1967) experiment also indicated that the magnitude of the SimNCE, and presumably the magnitude of frustration, was greatest at the point in space/time at which the $\mathrm{S}$ - cue was first introduced.

A similar explanation can be applied to SucNCEs. Spence (1956) indicated agreement with Crespi (1944) that the depression effect was probably due to disruptive emotional responses occurring subsequent to a decrease in reward magnitude. The emotional responses disrupted behavior by producing responses that competed with the performance of the instrumental task. Again, Amsel's (1962) frustration concept has been identified with these presumed emotional responses (e.g., Cleland et al., 1969; Spear \& Spitzner, 1966).

A source of indirect support for this line of reasoning may be derived from the effects of drugs on contrast. The data reviewed above indicate that 
drugs that have anxiolytic effects in humans (i.e., amobarbital, ethanol, and chlordiazepoxide) also tend to reduce negative contrast. The study by Ridgers and Gray (1973) is especially interesting in this regard, since the barbiturate drug reduced negative contrast but had no effect on positive contrast. Of course, the drugs used in these experiments have widespread effects in the nervous system, and whether it is fruitful to regard the contrastameliorating effects of the drug as being due to the reduction of an emotional response similar to those experienced by humans remains moot.

Two other complications in interpreting the effects of drugs on contrast arise when the successive and simultaneous data from sucrose studies are considered. In the relevant section above, it was mentioned that various anxiolytic drugs seem to influence successive negative contrast only under restricted conditions, and that these same drugs seem to have no tendency to reduce simultaneous contrast.

Another source of support for the hypothesis that emotional responses are involved in contrast comes from the experiment of Goldman, Coover, and Levine (1973). These investigators found an increased release of adrenocorticosteroid hormones in a behavioral negative-contrast experiment. A release of steroid hormones also occurs following a shift to extinction (Dantzer, Arnone, \& Mormede, 1980), a shift that may be considered the limiting case of negative contrast (Gonzalez \& Bitterman, 1960; Gonzalez et al., 1973). Thus, complete or incomplete reductions in reward activate a component of the sympathetic response to stress.

Further circumstantial support comes from studies of downshifts in sucrose reward in an open-field context. Such a shift leads large increases in activity, both ambulation and rearing behaviors (Flaherty et al., 1978; Flaherty, Ciszewski, \& Kaplan, 1979; Flaherty, Powell, \& Hamilton, 1979) which, in mice, have been described as factors loading on an emotional reactivity dimension (Royce, Poley, \& Yeudall, 1973).

Some interesting support for an emotionality interpretation is derived from two studies which investigated the relationship between other aversive situations and NCEs. Shanab and Ferrel (1975) showed that prior NCE experience ameliorated the effects of delay of reward on behavior, and prior experience with delay of reward reduced the effects of a reward downshift. Since there is evidence that delay of reward may be considered aversive (e.g., Daly, 1974a), the transfer between delay and NCE could reasonably be considered to be due to a common aversiveness.

Rosellini and Terris (1975) showed, in three experiments, that animals trained to persist under conditions of shock punishment did not show a NCE when reward was shifted downward. Indeed, the behavior of these rats did not change at all when reward was decreased. This transfer is consistent with the idea that punishment and decreased reward share similar emotional properties (e.g., Wagner, 1969). Rosellini and Terris also showed that if rats were sensitized to shock in an apparatus different from the one in which reward was to be reduced (but not trained to persist to shock), then a SucNCE did occur when reward was downshifted and, in fact, was slightly larger in the shocked animals. This result is also consistent with the assumption that electric shock and downshifts in reward have some common, perhaps emotional, properties.

In addition to support from the indirect evidence listed above, the frustration explanation of negative contrast effects is appealing both because many of the variables influencing the intervening response of frustration have been worked out in other contexts (e.g., Amsel, 1962, 1967) and because the frustration interpretation can incorporate many of the negative contrast findings. For example, the reward disparity effect can be accounted for by assuming that the larger the preshift or S+ reward magnitude, the greater is the frustration produced by the postshift or S- reward. This account of negative contrast is also consonant with the frustration account of the effects of amount of reward on resistance to extinction (Wagner, 1961). The theory can also account for the effects of deprivation on negative contrast; the greater the deprivation, the more frustration elicited by the small reward. These results are consistent with the effects of deprivation on the Amsel frustration effect: a larger frustration effect is obtained with more extreme deprivation (Dunlap \& Frates, 1970). Some effects of prior experience with differential conditioning or with a partial reinforcement schedule (especially those found by Mikulka et al., 1967) can also be interpreted in frustration terms. The explanation here is that both of these learning situations involve frustration and eventually lead to the conditioning of the stimulus component of anticipatory frustration, $S_{f}$, to the instrumental response. Because of this conditioning, the frustration experienced later in the successive contrast paradigm does not have a disruptive effect on instrumental performance. This explanation is consistent with the frustration explanation of the partial reinforcement extinction effect itself.

This interpretation also makes contact with applications of the frustration or emotionality concept in human social and clinical situations and, especially, to a theory of incentive loss and depression in humans (Klinger, 1975, 1977; Klinger, Barta, \& Kemble, 1974).

However, there are several aspects of the contrast situation that frustration theory has a difficult time accounting for. First, the effects of prior experience with partial reinforcement may only delay rather 
than eliminate a SucNCE (see above). A frustration explanation of this delay would seem to require some additional elaboration of the theory. The theory is also not equipped at the present time to handle the effects of the sequential variables reported above. A third difficulty that frustration theory has is predicting the duration of contrast effects. Emotionally based theories generally imply that contrast effects should be of relatively short duration. Within the context of frustration theory, negative contrast effects would be expected to diminish either because of a reduction in primary frustration response, $R_{F}$, or because anticipatory frustration, $r_{f}-s_{f}$, has been conditioned to the instrumental response. This latter mechanism is probably more consistent with other developments in the theory. SucNCEs generally are of short duration (E. J. Capaldi \& Lynch, 1967; Flaherty et al., 1973; McHose \& Moore, 1978; Vogel et al., 1968), but there are exceptions (e.g., DiLollo, 1964a). On the other hand, SimNCEs are often of long duration and sometimes give no indication of dissipating by the end of the experiment (Bower, 1961; Ludvigson \& Gay, 1967; Flaherty et al., 1977).

Crespi's interpretation of PCEs as being due to a positive emotion such as elation, joy, or eagerness (Crespi, 1942, 1944) has not been systematically examined in a way at all corresponding to the concept of frustration. However, given that there are verbal reports of subjective experience in humans that seems to correspond to Crespi's idea of energized behavior brought about by the occurrence of something better than expected (e.g., Sheehan, 1978, pp. 210-211), such a systematic examination might be worth the effort.

\section{Exploration}

Elliott (1928) suggested that the negative-contrast effect obtained in measures of maze behavior (time and errors) when rats were downshifted in reward quality represented exploratory responses on the part of the animals. There has been very little research on this topic. Flaherty and his colleagues (Flaherty et al., 1978; Flaherty, Ciszewski, \& Kaplan, 1979; Flaherty, Powell, \& Hamilton, 1979) downshifted sucrose concentration in an open field and presented additional sources of sucrose at novel locations during the postshift period. The downshift led to reliable increases (and contrast effects) in ambulation and rearing behavior, but there was no clear evidence of systematic exploration. However, the open field may not be the optimum apparatus for testing Elliott's hypothesis. Perhaps and apparatus such as the Olton sunburst maze would yield a different picture (Olton \& Samuelson, 1978).

\section{Sensory Processes}

The applicability of adaptation level, a concept derived from sensory-perceptual research, to incentive contrast was considered earlier. In this section, we will briefly consider one situation in which taste processes might be directly involved in contrast obtained in consummatory behavior.

As previously described, SimCEs obtained in the consumption of sucrose solutions are symmetrical, robust, and enduring (Flaherty \& Largen, 1975). Also, as previously described, an analysis of contrast obtained when different pairs of sucrose solutions were presented showed that a change in the ratio of lick frequencies to solution pairs is exponentially related to the ratio of concentration pairs. The exponent is approximately 1.15 (Flaherty \& Kaplan, 1979; Flaherty \& Sepanak, 1978), a value within the range obtained in human judgments of relative sweetness (Meiselman, 1917; Moskowitz, 1970; Stevens, 1969). Furthermore, these contrast effects are uninfluenced by a variety of treatments that do influence SucNC, treatments such as drugs and disinhibitory stimuli. These contrast effects are, however, reduced if the animals drink water between experiencing the two solutions (Flaherty, Checke, \& Dodgen, Note 5). It is our interpretation that these contrast effects are mediated principally by peripheral sensory processes much like those demonstrated in human taste by Bartoshuk (1968) and McBurney (1972).

\section{Summary of Explanatory Concepts}

In summarizing attempted explanations of contrast, two caveats must be considered. First, the various theoretical models reviewed above are not all mutually exclusive, and it could well be that more than a few of them taken together will be involved in the eventual understanding of contrast. Second, it is also possible that all apparent contrast effects do not represent the same phenomenon, and that there are different explanations for different types of contrast.

Let us consider the first possibility. A shift from large to small reward may engender at least a momentary generalization decrement or neophobic response. Following the detection that the current reward is different from the memory of previous rewards (Spear, 1967), there may be an evaluation process. This evaluation is based on a comparison of the current reward with the memory of the average reward (adaptation level, reinforcement level) received in that or similar contexts (transsituational contrast) in the past. If the result of the evaluation is that the current reward is substantially inferior to the average to be expected in that context, a stress system may be activated (as is the case in extinction; Dantzer et al., 1980), and there may result behavioral manifestations of this emotional state. Concomitants of this emotional state could include the active inhibition of approach to the new reward and the elicitation of competing and/or exploratory behaviors.

Now, with regard to the second caution, behaviorally similar contrast effects occur in runway situations, in consummatory response situations, and in operant tasks. However, it is possible that the underlying mechanisms are different in at least some of these 
situations. For example, in this review some evidence has been presented which indicated that SimCEs in the consumption of sucrose solutions might be produced by a mechanism (sensory processes) different from the successive contrast effects in the consumption of sucrose solutions (emotional processes?). There is clearly much work to be done.

\section{SUMMARY AND CONCLUSIONS}

(1) The major empirical relations reviewed in this paper are summarized in Table 1.

(2) The evidence in favor of PCEs, particularly SucPCEs, is substantial. The use of constant delay of reward or long runways, or shifting reward upward while the animals are still showing a SucNCE from a previous downshift, all seem to contribute to the occurrence of SucPCEs. Shifting after few acquisition trials or giving prior experience with the large reward seems to be ineffective in promoting SucPCEs. The role of deprivation is uncertain. The occurrence of SimPCEs is also favored by use of a constant delay of reward and, possibly, by conditions which reduce uncertainty as to which reward (large or small) will occur on a given trial. PCEs are obtained in other situations, such as consummatory responding for sucrose or with shifts in reward percentage, as well as with reward magnitude shifts.

(3) It is not certain whether constant delay contributes to the occurrence of PCEs simply because it reduces the "ceiling effect" problem or whether it also introduces an element of aversiveness which acts to enhance PCEs.

(4) Degree of NCEs, and possibly PCEs, varies directly with degree of reward disparity, and, if varied reward is experienced preshift, CEs are likely to be related to the average preshift reward.

(5) Both PCEs and NCEs seem to occur more readily with shifts in percentage of reward than with shifts in delay of reward.

(6) The limits to which contrast effects are transsituational have not been explored.

(7) SucNCEs in runway behavior seem to develop when rats are about 25 days old-later than the partial reinforcement extinction effect.

(8) Analysis of contrast from a comparative perspective indicates that SimCEs and behavioral contrast effects vary together and are different from SucCEs.

Table 1

Effects of Indicated Variables on Successive and Simultaneous Negative and Positive Contrast

\begin{tabular}{|c|c|c|c|c|}
\hline & \multicolumn{4}{|c|}{ Type of Contrast } \\
\hline & SucNCE & SucPCE & SimNCE & SimPCE \\
\hline \multicolumn{5}{|l|}{ Variable: } \\
\hline Reward Disparity & $\uparrow$ & $\uparrow$ & $\uparrow$ & $?$ \\
\hline Degree of Deprivation & $\uparrow$ & $?$ & $?$ & $?$ \\
\hline Intertrial Interval & $\downarrow$ & $?$ & $\downarrow$ & $?$ \\
\hline Retention Interval & $\downarrow$ & $?$ & $?$ & ? \\
\hline \multicolumn{5}{|l|}{ Prior Experience With: } \\
\hline Large Reward & $\uparrow$ & $\uparrow-$ & $?$ & $?$ \\
\hline Small Reward & $\downarrow ?$ & $?$ & $\downarrow ?$ & $?$ \\
\hline Partial Reward & $\downarrow ?$ & - & - & - \\
\hline Varied Reward & $\downarrow$ & $?$ & $?$ & $?$ \\
\hline \multicolumn{5}{|l|}{ Occurs With Shifts in: } \\
\hline Delay & No? & No & Yes & $?$ \\
\hline Percentage & Yes & Yes & $?$ & No \\
\hline \multicolumn{5}{|l|}{ Sucrose } \\
\hline Runway & No & $?$ & Yes & No \\
\hline Consummatory & Yes & Yes? & Yes & Yes \\
\hline Aversive Stimuli & Yes & $?$ & $?$ & $?$ \\
\hline \multicolumn{5}{|l|}{ Influence of Drugs: } \\
\hline Amobarbital & $\downarrow$ & $?$ & $?$ & $-?$ \\
\hline Chlordiazepoxide & $\downarrow$ & $?$ & $?$ & $?$ \\
\hline Chlorpromazine & - & $?$ & $?$ & $?$ \\
\hline Scopolamine* & - & $?$ & - & - \\
\hline Imipramine* & $?$ & $?$ & - & - \\
\hline Ethyl alcohol* & $\downarrow$ & $?$ & $?$ & $?$ \\
\hline \multicolumn{5}{|l|}{ Influence of Lesions: } \\
\hline Hippocampal Lesions & $-?$ & $?$ & $?$ & $?$ \\
\hline Cingulate Lesions & $\downarrow$ & $?$ & $?$ & $?$ \\
\hline Septal Lesions* & - & $?$ & $?$ & $?$ \\
\hline
\end{tabular}

Note-An upward arrow ( $\uparrow)$ indicates that increases in the variable enhance contrast. A downward arrow ( 1 ) indicates that increases in the variable diminish contrast. A hyphen $(\cdot)$ indicates that there was no effect of the variable. A question mark (?) indicates that the variable was not investigated or the results were uncertain; combined with another symbol, a question mark indicates that what is shown was the tendency of the results, but the results were uncertain. *Investigated in sucrose consummatory response paradigm. 
(9) PCEs and NCEs occur readily in the consumption of sucrose, and SimNCEs occur in runway behavior with a sucrose reward, but SucNCEs do not occur with downshifts in sucrose in the runway.

(10) A period of absence of saccharin, quinine, or ethanol leads to an apparent PCE when these substances are returned. This contrast may be related to PCEs that occur in instrumental behavior when a period of nonreward is interpolated between reward sessions.

(11) Sensory-perceptual theories have little success in explaining contrast, except in the case of some SimCE data obtained in the consumption of sucrose solutions.

(12) There is substantial support for a motivationalemotional interpretation of contrast, yet theorizing in this area remains at a relatively low formal level and few precise predictions can be generated.

(13) Associative explanations of contrast have been developed the furthest, in formal terms, yet, aside from some role for generalization decrement in SucNCEs and the clear usefulness of an incentive averaging or reinforcement-level concept, there is no substantial support for these theories.

(14) There has been very little research regarding the possibility that a contrast experience generates exploratory behavior.

(15) Anxiolytics such as chlordiazepoxide, sodium amobarbital, and ethanol reduce SucNCEs.

(16) Very little is known about the neural or neurochemical events correlated with contrast.

\section{REFERENCE NOTES}

1. Sparling, D. L., \& Capaldi, E. J. The negative contrast effect as a function of trial spacing. Paper presented at the meeting of the Midwestern Psychological Association, 1972.

2. Flaherty, C. F., Becker, H. C., Checke, S., \& Kalucki, B. Negative contrast obtained with repeated shifts in sucrose concentration. Manuscript submitted for publication.

3. Capaldi, E. J. Reinforcement level: An expectancy-associative approach to relative reinforcement and nonreinforcement effects. Paper presented at the Arlington Symposium on Learning, Arlington, Texas, 1975.

4. Spear, N. E. Contrast effects of reinforcer magnitude and within-subject effects of other reinforcement conditions. In Empirical and theoretical problems in the analysis of shifts in reinforcement parameters. Symposium presented at the meeting of the Midwestern Psychological Association, Chicago, 1968.

5. Flaherty, C. F., Checke, S., \& Dodgen, C. Simultaneous gustatory contrast reduced by intervening water consumption. Manuscript in preparation.

\section{RTFLRENCES}

Amsel, A. Frustrative nonreward in partial reinforcement and discrimination learning. Psychological Review, 1962, 69, 306-328.

Amsel, A. Partial reinforcement effects on vigor and persistence. In K. W. Spence J. T. Spence (Eds.), The psychology of learning and motivation: Advances in research and theory (Vol. 1). New York: Academic Press, 1967.
AMseL, A., \& Rousgend, J. Motivational properties of frustration. Journal of Experimental Psychology, 1952, 43, 363-368. Ashida, S., \& Birch, D. The effects of incentive shift as a function of training. Psychonomic Science, 1964, 1, 201-202.

Ashton, A. B., Gandelman, R., \& Trowill, J. A. Effects of food deprivation upon elation of saccharin drinking following a temporary shift to water. Psychonomic Science, 1970, 21, 5-6. (a)

Ashton, A., Gandelman, R. J., \& Trowill, J. A. Effects of reinforcement shifts upon subsequent sucrose consumption. Psychonomic Science, 1970, 21, 7-8. (b)

Ashton, A. B., \& Trowilt, J. A. Effects of reinforcement shifts upon lick rate. Psychonomic Science, 1970, 21, 8-10.

Atrens, D. M., Von Vietinghopf-Riesch, F., \& DerKarabetian, A. Reinforcement contrast effect on the rewarding and aversive components of intracranial stimulation. Learning and Behavior, 1973, 4, 397-404.

BacotTi, A. V. Home cage feeding time controls responding under multiple schedules. Animal Learning \& Behavior, 1976, 4, 41-44.

Baltzer, V., \& Weiskrantz, L. Negative and positive behavioral contrast in the same animals. Nature, 1970, 228, 581-582.

Barnes, W., \& Tombaugh, T. Another failure to obtain negative contrast following reductions in sucrose reward. Psychological Reports, 1973, 33, 801-802.

BARnetT, S. A. The rat: A study in behavior. London: Methuen, 1963.

Bartoshuk, L. M. Water taste in man. Perception \& Psychophysics, 1968, 3, 69-72.

Becker, H. C., \& Flaherty, C. F. Influence of ethanol on contrast in consummatory behavior. Psychopharmacology, 1982, 77, 253-258.

BEERY, R. G. A negative contrast effect of reward delay in differential conditioning. Journal of Experimental Psychology, 1968, 77, 429-434.

Benefield, R., Oscos, A., \& Ehrenfreund, D. Role of frustration in successive positive contrast. Journal of Comparative and Physiological Psychology, 1974, 86, 648-651.

Bevan, W. The pooling mechanism and the phenomena of reinforcement. In O. J. Harvey (Ed.), Motivation and social interaction. New York: Ronald, 1963.

Bevan, W. An adaptation-level interpretation of reinforcement. Perceptual and Motor Skills, 1966, 23, 511-531.

Bevan, W. The contextual basis of behavior. American Psychologist, 1968, 23, 701-713.

Bindra, D. A unified account of classical conditioning and operant training. In A. H. Black \& W. F. Prokasy (Eds.), Classical conditioning II. New York: Appleton-Century-Crofts, 1972.

Bindra, D. A motivational view of learning, performance and behavior modification. Psychological Review, 1974, 81, 199-213.

BiNTz, J. Between and within-subject effect of shock intensity on avoidance in goldfish (Carassius auratus). Journal of Comparative and Physiological Psychology, 1971, 75, 92-97.

BitTeruan, M. E. The comparative analysis of learning. Science, 1975, 188, 699-709.

Bitterman, M. E. Incentive contrast in honey bees. Science, $1976,192,380-382$.

Black, R. W. Shifts in magnitude of reward and contrast effects in instrumental and selective learning: $A$ reinterpretation. Psychological Review, 1968, 75, 114-126.

Bohdanecky, Z., J Jarvix, M. E. Impairment to one-trial passive avoidance learning in mice by scopolamine, scopamine methylbromide and physostigmine. International Journal of Neuropharmacology, 1967, 6, 217-222.

Boures, R. C. Reinforcement, expectancy, and learning. Psychological Review, 1972, 79, 394-409.

Bower, G. H. A contrast effect in differential conditioning. Journal of Experimental Psychology, 1961, 62, 196-199.

Bower, G. H., Fowler, H., \& Trapold, M. A. Escape learning as a function of amount of shock reduction. Journal of Experimental Psychology, 1959, 58, 482-484. 
Brownlee, A., \& Bitterman, M. E. Differential reward conditioning in the pigeon. Psychonomic Science, 1968, 12, 345-346.

Bunss, R. A. Effects of sequences of sucrose reward magnitudes with short ITIs in rats. Animal Learning \& Behavior, 1976, 4, 473-479.

Burns, R. A., \& Bunns, D. P. Reduction in sucrose reward magnitude without generalization decrement. Bulletin of the Psychonomic Society, 1978, 12, 196-198.

Burns, R. A., Dupres, E. S., \& Lonig, T. S. Successive reductions of liquid and solid rewards. Bulletin of the Psychonomic Society, 1978, 12, 351-354.

Burns, R. A., Woodward, W. T., Henderson, T. B., \& Bitterman, M. E. Simultaneous contrast in the goldfish. Animal Learning \& Behavior, 1974, 2, 97-100.

CALEF, R. S. The effect of large and small magnitudes of intertrial reinforcement on successive contrast effects. Psychonomic Science, 1972, 29, 309-312.

Calef, R. S., Calef, R. A., Maxwell, F. R., \& McHewitt, E. R. Positive discrimination contrast with delay of reward or low drive. Bulletin of the Psychonomic Society, 1975, 6, 120-122.

Calef, R. S., Calef, R. A., Prochaska, A. D., \& Geller, E. S. Negative contrast as a function of reinforcement location and consistent vs. varied reward magnitude. Bulletin of the Psychonomic Society, 1978, 12, 471-474.

Calet, R. S., Hopkins, D. C., McHewitt, E. R., \& MaXwell, F. R. Performance to varied reward following continuous reward training in the runway. Bulletin of the Psychonomic Society, $1973,2,103-104$.

Campbell, D. E., Crumbaugh, C. M., Knouse, S. B., \& SNodorass, E. A test of the "ceiling effect" hypothesis of positive contrast. Psychonomic Science, 1970, 20, 17-18.

Campbell, E. M., \& Meyer, P. A. Effects of daily reward sequence on simultaneous and successive negative contrast in rats. Journal of Comparative and Physiological Psychology, 1971, 74, 434-440.

Capaldi, E. D. Simultaneous shifts in reward magnitude and level of food deprivation. Psychonomic Science, 1971, 23, 357-359.

Capaldi, E. D. Effects of changing alley color on the successive negative contrast effect. Bulletin of the Psychonomic Society, $1978,12,69-70$

Capaldi, E. D., \& Sinah, R. Percentage body weight and the successive negative contrast effect in rats. Learning \& Motivation, 1973, 4, $405-416$.

Capaldi, E. D., Smith, N. S., \& White, L. A. Control of reward expectancies by drive stimuli. Journal of Experimental Psychology: Animal Behavior Processes, 1977, 3, 178-188.

Capaldi, E. J. Partial reinforcement: A hypothesis of sequential effects. Psychological Review, 1966, 73, 459-477.

Capaldi, E. J. Successive negative contrast effect: Intertrial interval, type of shift, and four sources of generalization decrement. Journal of Experimental Psychology, 1972, 96, 433-438.

Capaldr, E. J. Partial reward either following or preceding consistent reward: A case of reinforcement level. Journal of Experimental Psychology, 1974, 102, 954-962.

Capaldi, E. J., \& LYNCh, D. Repeated shifts in reward magnitude: Evidence in favor of an associational and absolute (noncontextual) interpretation. Journal of Experimental Psychology, 1967, 75, 226-235.

CaPaldi, E. J., \& Zirr, D. R. Schedule of partial reward and the negative contrast effect. Journal of Comparative and Physiological Psychology, 1969, 68, 593-596.

Caruton, P. L. Brain acetylcholine and inhibition. In J. T. Tapp (Ed.), Reinforcement and behavior. New York: Academic Press, 1969.

Chechile, R., \& Fowlen, H. Primary and secondary negative incentive contrast in differential conditioning. Journal of Experimental Psychology, 1973, 97, 189-197.

Chen, J., Gross, K., \& Am8et, A. Ontogeny of successive nega- tive contrast and its dissociation from other paradoxical effects in preweanling rats. Journal of Comparative and Physiological Psychology, 1981, 95, 146-149.

Ciszewski, W. A., \& Flaherty, C. F. Failure of a reinstatement treatment to influence negative contrast. American Journal of Psychology, 1977, 90, 219-229.

Cleland, E. A., Williams, M. Y., \& Dilollo, V. Magnitude of negative contrast effect in relation to drive level. Psychonomic Science, 1969, 15, 121-122.

Cochane, T. L., Scobie, S. R., \& Fallon, D. Negative contrast in goldfish (Carassius auratus). Bulletin of the Psychonomic Society, 1973, 1, 411-413.

Collien, G., \& MARx, M. H. Changes in performance as a function of shifts in the magnitude of reinforcement. Journal of $E x$ perimental Psychology, 1959, 57, 305-309.

Collier, G., KNarR, F. A., \& Marx, M. H. Some relations between the intensive properties of the consummatory response and reinforcement. Journal of Experimental Psychology, 1961, 62, 484-495.

Cowan, P. E., \& Barnett, S. A. The new-object and newplace reaction of Rattus rattus L. Zoological Journal of the Linnean Society, 1975, 56, 219-234.

Cox, W. M. A review of recent incentive contrast studies involving discrete-trial procedures. Psychological Record, 1975, 25, 373-393.

CaEsp1, L. P. Quantitative variation in incentive and performance in the white rat. American Journal of Psychology, 1942, 55, 467-517.

Crespi, L. P. Amount of reinforcement and level of performance. Psychological Review, 1944, 51, 341-357.

DALY, H. B. Excitatory and inhibitory effects of complete and incomplete reward reduction in the double runway. Journal of Experimental Psychology, 1968, 76, 430-438.

DALY, H. B. Reinforcing properties of escape from frustration. In $\mathrm{G}$. H. Bower (Ed.), The psychology of learning and motiva. tion. New York: Academic Press, 1974. (a)

DALY, H. B. Arousal of frustration following gradual reductions in reward magnitude in rats. Journal of Comparative and Physiological Psychology, 1974, 86, 1149-1155. (b)

Daly, H. B., \& Rosenbera, K. M. Infantile stimulation and its effects on frustration- and fear-motivated behavior in rats. Learning and Motivation, 1973, 4, 381-396.

Dantzer, R., Arnone, M., \& Mormede, P. Effects of frustration on behavior and plasma corticosteroid levels in pigs. Physiology \& Behavior, 1980, 24, 14.

DAVENPORT, J. $\boldsymbol{W}$. The interaction of magnitude and delay of reinforcement in spatial discrimination. Journal of Comparative and Physiological Psychology, 1962, 55, 267-273.

Davis, S. F., Harper, W. E., \& Seaco, J. D. Runway performance of normal, sham, and anosmic rats as a function of magnitude of reward and magnitude shift. Bulletin of the Psychonomic Society, 1975, 6, 367-369.

Davis, S. F., \& NorTh, A. J. The effect of varied reinforcement training on behavior following incentive reduction. Psychonomic Science, 1967, 9, 395-396.

Denenberg, V. H., \& Grota, L. J. Social-seeking and noveltyseeking behavior as a function of differential rearing histories. Journal of Abnormal and Social Psychology, 1964, 69, 453-456.

DiLozio, V. Runway performance in relation to runway-goal-box similarity and changes in incentive amount. Journal of Comparative and Physiological Psychology, 1964, 58, 327-329. (a)

DiLollo, V. Contrast effects in the judgement of lifted weights. Journal of Experimental Psychology, 1964, 68, 383-387. (b)

DiLollo, F. D., \& BeEz, V. Negative contrast effect as a function of magnitude of reward decrement. Psychonomic Science, $1966,5,99-100$.

DiLollo, V., \& MEYER, P. N. Variations in fluid intake following shifts between water and saccharin solution. Psychonomic Science, 1970, 18, 55-56. 
Dubs, R., Ashton, A. B., Trowill, J. A. Responses to palatability shifts: Effects of varying the retention interval. Psychonomic Science, 1970, 21, 10-12.

Dunkay, P. J. Contrasted conditions of reinforcement: $\boldsymbol{A}$ selective critique. Psychological Bulletin, 1968, 69, 295-315.

Dunнaи, P. J., KILPs, B. Shifts in magnitude of reinforcement: Confounded factors or contrast effects. Joumal of Experimental Psychology, 1969, 79, 373-374.

Dunlap, W. P., Frates, S. D. Influence of deprivation on the frustration effect. Psychonomic Science, 1970, 21, 1-2.

Dycx, D. G., Dregel, K. M., Thicesen, R. B., Dilollo, V. Extinction following separate-phase acquisition: Effects of shifts in reinforcement percentage and N-length. Bulletin of the Psychonomic Soclety, 1977, 10, 439-412.

Ehasmfarund, D. Effect of drive on successive magnitude shift in rats. Journal of Comparattve and Physiological Psychology, $1971,76,418-423$

Ehrenfreund, D., \& BAd1A, P. Reaponse strength as a function of drive level and pre- and postshift incentive magnitude. Journal of Experimental Psycholozy, 1962, 63, 468-471.

Eraengenger, R., Frank, M., \& Park, D. C. Incentive contrast of choice behavior. Journal of Experimental Psychology: Animal Behavior Processes, 1979, 4, 346-354.

Elliotr, M. H. The effect of change of reward on the maze performance of rats. University of Callfornia PublicationsPsychology, 1928, 4, 19-30.

Fagen, J. W. \& Shotmarem, G. E. Contrast effects in the rat: A developmental study. Developmental Psychoblology, 1979, 12, 83-92.

Fenez, H. J., \& Shanab, M. E. Contrast effects as a function of shifts in delay of water reward. Bulletin of the Psychonomic Society, 1975, 5, 417-420.

Fitzozkald, F. S. K. The great Gatsby. New York: Scribner, 1953.

Flaheaty, C. F., \& Avdexd, A. Bidirectional contrast as a function of rate of alternation of two sucrose solutions. Bulletin of the Psychonomic Soclety, 1974, 4, s05-507.

Frakerty, C. F., \& Avdees, A. Transoituational negative contrast. Animal Learning \& Behavior, 1976, 4, 49-52.

Flakenty, C. F., Beceer, H. C., Deiscoll, C. D. Conditions under which amobarbital sodium influences contrast in consummatory behavior. Physlological Pyychology, 1982, 10, 122-128.

Funerty, C. F., Blitzer, R., \& Collise, G. H. Open field behaviors elicited by reward reduction. American Journal of Psychology, 1978, 91, 429-443.

Flaherty, C. F., Capobianco, S., Hamilton, L. W. Effects of septal lesions on retention of negative contrast. Physlolozy \& Behavior, 1973, 11, 625-631.

Flahezty, C. F., \& Capkio, M. Dissociation between instrumental and consummatory measures of incentive contrast. American Journal of Psychology, 1976, 63, $485-498$.

Flahesty, C. F., Chrcke, S. Anticipation of Incentive gain. Animal Learning \& Behavior, 1982, 10, 177-182.

Flaherty, C. F., Ciezewber, W. A., \& Kapuan, P. S. Retention of tuste quality following brief exposure to sucrose. Chemical Senses and Flavour, 1979, 4, 73-78.

Flaherty, C. F. De Discolc, C. Amobarbital sodium reduces successive gututory contrast. Psychopharmacology, 1980, 69, 161.162.

Frahenty, C. F., Hamilton, L. W. Reaponsivity to decreasing sucrose concentrations following septal lesions in the rat. Physiology \& Behavior, 1971, 6, 431-437.

Fuarerty, C. F., Kaplan, P. Gustatory contrast in rats. Chemical Senses and Flovour, 1979, 4, 63-72.

Flahemty, C. F., Kelly, J. Effect of deprivation state on successive negative contrast. Bulletin of the Psychonomic Society, 1973, 1, 365-367.

Flaherty, C. F., Lanore, J. Within-subjects positive and negative contrast effects in rats. Journal of Comparative and Physiological Psychology, 1975, 01, 653-664.

Flahenty, C. F., L Lomband, B. R. Effect of prior differential taste experience on retention of taste quality. Bulletin of the Psychonomic Society, 1977, 9, 391-394.

Flaherty, C. F., Lombandi, B. R., Kapuet, J., D'Auato, M. R. Incentive contrast undiminished by extended testing, imipramine, or chlordiazepoxide. Pharmacology, Blochemistry and Behavior, 1977, 7, 315-322.

Flahetet, C. F., Lombardi, B. R., Whighteon, J., \& Deptula, D. Conditions under which chlordiazepoxide influences gustatory contrast. Psychopharmacology, 1980, 67, 269-277.

Flaherty, C. F., \& Meinanth, A. B. Influences of scopolamine on sucrose intake under absolute and relative test conditions. Phystological Psychology, 1979, 7, 412-418.

Flaheety, C. F., Powrll, G., \& Hamilton, L. W. Septal lesion, sex, and incentive shift effects on open field behavior of rats. Physiology \& Behavior, 1979, 22, 903-909.

Flaherty, C. F., Riley, E. D., \& Spak, N. E. Effect of sucrose concentration and goal units on runway behavior in the rat. Learning and Motivation, 1973, 4, 163-175.

Flahenty, C. F., \& Sepanak, S. J. Bidirectional contrast, matching, and power functions oblained in sucrose consumption by rats. Anlmal Learning \& Behovior, 1978, 6, 313-319.

Flahenty, C. F., Thoncoso, B., \& Deschu, N. Open field behaviors correlated with reward availability and reward shift in three rat strains. American Journal of Psychology, 1979, 92 $385-400$.

Flahenty, C. F., Weiohtgon, J., Deptuli, D., \& Duston, C. Chlordinzepoxide does not influence simultaneous gustatory contrast. Bulletin of the PJychonomic Society, 1979, 14, 216-218.

Fox, P. A. Reward and nonreward produced stimuli and performance to patterned reinforcement. Journal of Comparative and Physiological Psycholozy, 1972, 80, 342-348.

Fox, P. A., Calef, R. S., Gavelex, J. R., \& McHose, J. H. Synthesis of differential conditioning and double alley data: Performance to $S+$ as a function of intertrial interval and antedating reward events. Psychonomic Science, 1970, 18, 141-143.

Franchina, J. J., \& Brown, T. S. Reward magnitude shift effects in rats with hippocampal lesions. Journal of Comparative and Physiological Pyycholozy, 1971, 76, 365-370.

Galmeitr, K. J., Rashorte, M. E., \& Amget, A. Withinsubjects partial reinforcement effects varying percentage reward to the partial stimulus between groups. Journal of Experimental Psycholosy, 1968, 77, 547-531.

Gandelyan, R., \& Trowill. J. A. Effects of reinforcement shifts upon subsequent saccharin consumption. Psychonomic Science, 1969, 15, 25.

Gavelex, J. R., \& McHoar, J. H. Contrast effects in differential delay of reward conditioning. Journal of Experimental Psychology, 1970, 86, 454-457.

Gleituan, H., Strimuan, F. Depression effect as a function of retention interval before and after shift in reward magnitude. Journal of Comparative and Physiological Psycholozy, 1964, 57, 158-160.

Goldyan, L., Coover, G. D., Levine, S. Bj-directional effects of reinforcement shifts on pituitury adrenal activity. Physlology \& Behavior, 1973, 10, 209-214.

Gonzalez, R. C., Bittenyan, M. E. Spaced-trials partial reinforcement effect as a function of contrast. Journal of Comparative and Physiological Psychology, 1969, 67, 94-103.

Gonzalez, R. C., \& Champlin, G. Positive behavioral contrant, negative simultaneous contrast and their relation to frustration in pigeons. Journal of Comparative and Phystological Psychol. ogy, 1974, 07, 173-187.

Gonealez, R. C., Fennhort, D., D David, F. G. Contrast, resistance to extinction, and forgetting in rats. Journal of Comparative and Physiological Psychology, 1973, 24, 562-571.

Gonzalez, R. C., Frziy, M., \& Powres, A. B. The adjustment 
of goldfish to reduction in magnitude of reward in massed trials. Animal Learning \& Behavior, 1974, 2, 23-26.

Gonzalez, R. C., Gleitman, H., \& Bitterman, M. E. Some observations on the depression effect. Journal of Comparative and Physiological Psychology, 1962, 55, 578-581.

Gonzalez, R. C., Holmes, N. K., \& Bitterman, M. E. Resistance to extinction in the goldfish as a function of frequency and amount of reward. American Journal of Psychology, 1967, 80, 269-275.

Gonzalez, R. C., Potts, A., Pitcoff, K., \& Bitterman, M. E. Runway performance of goldfish as a function of complete and incomplete reduction in amount of reward. Psychonomic Science, 1972, 27, 305-307.

Gonzalez, R. C., \& Powers, A. S. Simultaneous contrast in goldfish. Animal Learning \& Behavior, 1973, 1, 96-98.

Goodrich, K. P., \& ZARETsKY, H. Running speed as a function of concentration of sucrose during pretraining. Psychological Reports, 1962, 11, 463-468.

Gordon, W. C., Flaherty, C. F., \& Riley, E. P. Negative contrast as a function of the interval between preshift and postshift training. Bulletin of the Psychonomic Society, 1973, 1, 25-27.

Gunowitz, E. M., Rosen, A. J., \& Tessel, R. E. Incentive shift performance in cingulectomized rats. Journal of Comparative and Physiological Psychology, 1970, 70, 476-481.

HАGaвLOOM, S. J. Effects of a 24-hour intertrial interval on successive differential conditioning and simultaneous negative contrast. American Journal of Psychology, 1979, 92, 537-546.

Harris, S. J., Smith, M. G., \& Weinstock, S. Effects of nonreinforcement on subsequent reinforced running behavior. Journal of Experimental Psychology, 1962, 64, 388-392.

HARKer, G. 3. Delay of reward and performance of an instrumental response. Journal of Experimental Psychology, 1956, 51, 303-310.

Hearst, E., \& Peterson, G. B. Transfer of conditioned excitation and inhibition from one operant response to another. Journal of Experimental Psychology, 1973, 99, 360-368.

Helson, H. Adaptation-level theory: An experimental and systematic approach to behavior. New York: Harper \& Row, 1964.

Henderson, $\mathbf{K}$. Within-subjects partial reinforcement effects in acquisition and in later discrimination learning. Journal of Experimental Psychology, 1966, 72, 704-713.

Homzie, M. J., \& Ross, L. E. Runway performance following a reduction in the concentration of a liquid reward. Journal of Comparative and Physiological Psychology, 1962, 55, 1029-1033.

HoyenaA, K. T., \& HoyenaA, K. B. Experimental factors in the enhancement of saccharin intake after shifts to water. Animal Learning \& Behavior, 1973, 1, 244-246.

Huano, I.-N. Successive contrast effects as a function of type and magnitude of reward. Journal of Experimental Psychology, $1969,82,64-69$.

Hull, C. L. Principles of behavior. New York: Appleton-CenturyCrofts, 1943.

Hull, C. L. A behavior system. New Haven: Yale University Press, 1952.

Hulse, S. H. Reinforcement contrast effects in rats following experimental definition of a dimension of reinforcement magnitude. Journal of Comparative and Physiological Psychology, 1973, 85, 160-170.

Ison, J. R., Glas8, D. H., \& Daly, H. B. Reward magnitude changes following differential conditioning and partial reinforcement. Journal of Experimental Psychology, 1969, 81, 81-88.

Ison, J. R., \& KRANE, R. V. Induction in differential instrumental conditioning. Journal of Experimental Psychology, 1969, 80, 183-185.

Ison, J. R., \& Northman, J. Amobarbital sodium and instrumental performance changes following an increase in reward magnitude. Psychonomic Science, 1968, 12, 185-186.
Kalish, H. I., \& Haber, A. Prediction of discrimination from deprivation level. Journal of Comparative and Physiological Psychology, 1965, 60, 125-128.

KLINGER, E. Consequences of commitment to and disengagement from incentives. Psychological Review, 1975, 82, 1-25.

KLINGER, E. Meaning and void: Inner experience and the incentives in people's lives. Minneapolis: University of Minnesota Press, 1977.

Klinger, E., Barta, S. G., \& Kemble, E. D. Cyclic activity changes during extinction in rats: $A$ potential model of depression. Animal Learning \& Behavior, 1974, 2, 313-316.

KNaRR, F. A., \& Collier, G. Taste and consummatory activity in amount and gradient of reinforcement functions. Journal of Experimental Psychology, 1962, 63, 579-588.

Kramarcy, N., Mikulka, P., \& Freeman, F. The effects of dorsal hippocampal lesions on reinforcement shifts. Physiological Psychology, 1973, 1, 248-250.

Krane, R. V., \& Ison, J. R. Positive induction in differential instrumental conditioning: Effect of extended acquisition training. Journal of Comparative and Physiological Psychology, $1970,73,334-340$.

Krane, R. V., \& Ison, J. R. Positive induction in differential instrumental conditioning: Ef fect of the interstimulus interval. Journal of Comparative and Physiological Psychology, 1971, 75, 129-135.

Lehr, R. Partial reward and positive contrast effect. Animal Learning \& Behavior, 1974, 2, 221-224.

Leung, C. M., \& Jensen, G. D. Shifts in percentage of reinforcement viewed as changes in incentive. Journal of Experimental Psychology, 1968, 76, 291-296.

Lixely, D., LitTLE, L., \& MAckintosh, N. J. Extinction as a function of magnitude and percentage of food or sucrose reward. Canadian Journal of Psychology, 1971, 25, 130-137.

LoGAN, F. A. The role of delay of reinforcement in determining reaction potential. Journal of Experimental Psychology, 1952, 43, 393-399.

LoanN, F. A. Incentive. New Haven: Yale University Press, 1960.

LOMBARDI, B. R. Enhanced neophobia induced by incentive contrast. Unpublished doctoral dissertation, Rutgers University, 1978.

LOMBARDI, B. R. Enhanced neophobia induced by incentive contrast. Animal Learning \& Behavior, 1980, 8, 617-620.

Lombardi, B. R., \& Flaherty, C. F. Apparent disinhibition of successive but not of simultaneous negative contrast. Animal Learning \& Behavior, 1978, 6, 30-42.

Lowes, G., \& BitTe rman, M. E. Reward and learning in the goldfish. Science, 1967, 157, 455-457.

Ludviason, H. W., \& GAY, S. E. Differential reward conditioning, $S$ - contrast as a function of the magnitude of S+. Psy. chonomic Science, 1966, 5, 289-290.

Ludviason, H. W., \& GAY, R. A. An investigation of conditions determining contrast effects in differential reward conditioning. Journal of Experimental Psychology, 1967, 75, 37-42.

Mackinnon, J. R. Competing responses in a differential magnitude of reward discrimination. Psychonomic Science, 1968, 12, 333-334.

Mackintosh, N. J. Reward and the after-effects of reward in the learning of goldfish. Journal of Comparative and Physiological Psychology, 1971, 76, 225-232.

Mackintosh, N. J., \& LoRd, J. Simultaneous and successive contrast with delay of reward. Animal Learning \& Behavior, 1973, 1, 283-286.

MarX, M. H. Positive contrast in instrumental learning from qualitative shift in incentive. Psychonomic Science, 1969, 16, 254-255.

Matsumoto, R. T. Relative reward effects in differential conditioning. Journal of Comparative and Physiological Psychology, 1969, 68, 589-592.

Maxweld, F. R., Calef, R. S., Muraay, D. W., Shepard, J. C., 
\& Nonvilur, R. A. Positive and negative successive contrast effects following multiple shifts in reward magnitude under high drive and immediate reinforcement. Animal Learning \& Behavior, 1976, 4, 480-484.

McAllister, D. E., McAllister, W. R., Brooks, C. I., \& Goldman, J. A. Magnitude and shift of reward in instrumental aversive learning in rats. Journal of Comparative and Physiological Psychology, 1972, 80, 490-501.

McBurney, D. H. Gustatory cross adaptation between sweettasting compounds. Perception \& Psychophysics, 1972, 11, 225-227.

McCain, G., Boodes, A., \& LoBe, M. Extended training: Delay of reward. Bulletin of the Psychonomic Society, 1977, 9, 111-112.

McCain, G., \& Cooney, J. PCE: I. The effects of three reward magnitude shifts. Bulletin of the Psychonomic Society, 1975, 6, 523-526.

McCain, G., Lobb, M., Alyand, W., \& Leck, D. Delay of reinforcement: Extended training and multiple shifts. Bulletin of the Psychonomic Society, 1976, 7, 539.541.

McCain, G., Lobs, M., Newberry, J. Extended training and multiple shifts: Percentage of reward. Bulletin of the Psychonomic Society, 1976, 8, 191-193.

McHewirT, E. R. A comparison of S+ and S- depression effects in differential conditioning. Bulletin of the Psychonomic Society, $1974,3,3-5$.

McHewit, E. R. The effect of prior reward magnitude on the successive negative contrust effect. Bulletin of the Psychonomic Soclety, 1975, 6, 126-128.

McHewitt, E. R. Caler, R. S., Maxwell, F. R., Meyer, P. A., \& McHose, J. H. Synthesis of double alley and discrimination phenomena: Apparent positive S+ contrast in differential conditioning. Psychonomic Science, 1969, 16, 137-139.

McHose, J. H. Relative reinforcement effects: $S_{1} / S_{2}$ and $S_{1} / S_{1}$ paradigms in instrumental conditioning. Psychological Review, $1970,77,135-146$.

McHoss, J. H. Role of frustration in the development of relative and absolute $\mathbf{S}$ - discrimination contrast effects. Journal of Experimental Psychology, 1969, 81, 256-260.

McHose, J. H. Stimuli and incentives as determinants of the successive negutive contrast effect. Bulletin of the Psychonomic Society, 1973, 1, 264-266.

McHose, J. H., \& Howard, G. S. Performance in differential instrumental conditioning with infrequent $\mathbf{S}+$ presentations. Bulletin of the Psychonomic Society, 1973, 1, 132-134.

McHose, J. H., McHewitr, E. R., \& Peters, D. P. Average reward as a determinant of $\mathbf{S}$ - performance in differential conditioning. Psychonomic Science, 1972, 29, 129-132.

McHose, J. H., \& Moors, J. N. Expectancy, salience, and habit: A noncontextual interpretation of the effects of changes in the conditions of reinforcement on simple instrumental responses. Psychological Review, 1976, 83, 292-307.

McHose, J. H., \& Moonk, J. N. A comparison of positive and negative contrast effects. Bulletin of the Psychonomic Society, 1978, 11, 363-366.

Mchose, J. H., \& Peters, D. P. Differential instrumental conditioning as a function of percentage and amount of positive stimulus reward. Journal of Experimental Psychology, 1973, $100,413-415$

McHose, J. H., \& Perers, D. P. Partial reward, the negative contrast effect, and incentive averaging. Animal Learning \& Behavior, 1975, 3, 239-244.

McHose, J. H., \& Tauber, L. Changes in delay of reinforcement in simple instrumental conditioning. Psychonomic Science, 1972, 27, 291-292.

MeinRath, A. B. The role of neophobla in successive negative contrast: Effects of early handling and varied taste exposure. Unpublished doctoral dissertation, Rutgers University, 1980.

Meiselyan, H. L. Effect of presentation procedure on taste intensity functions. Perception \& Psychophysics, 1971, 10, $15-18$.

Mellones, R. L. Positive contrast in the rat as a function of number of preshift trials in the runway. Journal of Comparative and Physiological Psychology, 1971, 77, 329-336. (a)

Mellaren, R. L. Shift in magnitude of reward after minimal acquisition. Psychonomic Science, 1971, 23, 243-244. (b)

Mzllonen, R. L. Positive and negative contrast effects using delayed reinforcement. Learning \&otivation, 1972, 3, 185-193.

Mrulonen, R. L., \& Dyck, D. G. Reward magnitude in differential conditioning: Effects of sequential variables in acquisition and extinction. Journal of Comparative and Physiological Psychology, 1974, 86, 1141-1148.

Mellanen, R. L., Seyeert, J. A., Wrather, D. M., \& Dyck, D. G. Preshift reward magnitude and positive contrast in the rat. American Journal of Psychology, 1973, 86, 383-387.

Mrllanen, R. L., Wrather, D. M., \& Dyck, D. G. Differential conditioning and contrast effects in rats. Journal of Comparative and Physiological Psychology, 1972, 80, 478-483.

MEYER, P. A., \& CAMPBEL, E. M. Role of daily reward sequences on S- discrimination contrast in rats. Journal of Comparative and Physiological Psychology, 1973, 82, 426-433.

Mixulxa, P. J., Lehr, R., \& Pavlik, W. B. Effect of reinforcement schedules on reward shifts. Journal of Experimental Psy. chology, 1967, 74, 57-61.

Mitcheld, D., Fairbanks, M., \& Laycock, J. D. Suppression of neophobia by chlorpromazine in wild rats. Behavioral Blology, 1977, 19, 309-323.

Moore, J. N., McHose, J. H. The effects of delay of reward on negative contrast effects associated with reductions in reward magnitude. Bulletin of the Psychonomic Society, 1975, 6 , 497.500.

Monrison, J. H., \& Porter, J. J. Magnitude of reward in selective learning. Psychonomic Science, 1965, 3, 531-532.

Moskowitz, H. R. Ratio scales of sugar sweetness. Perception \& Psychophysics, 1970, 7, 315-320.

Murphy, H. M., \& Brown, T. S. Effects of hippocampal lesions on simple and preferential consummatory behavior in the rat. Journal of Comparative and Physiological Psychology, 1970, 72, 404-415.

Nation, J. R., \& Durst, D. The effects of schedules of reinforcement and gradual or abrupt increases in reward magnitude on resistance to extinction. Bulletin of the Psychonomic Society. $1980,15,425-427$.

Nation, J. R., Mellaren, R. L., \& Wrather, D. M. Contrast effects with shifts in punishment level. Bulletin of the Psychonomic Soclety, 1975, 5, 167-169.

Nation, J. R., Roop, S. S., \& Dickinson, R. W. Positive contrast following gradual and abrupt increases in reward magnitude using delay of reinforcement. Learning Motivation, 1976, 7, 571-579.

Nation, J. R., Wrather, D. M., \& Melloren, R. L. Contrast effects in escape conditioning of rats. Journal of Comparative and Physiological Psychology, 1974, 86, 69-73.

Olton, D. S., \& Samuelson, R. J. Remembrance of places past: Spatial memory in rats. Journal of Experimental Psychology: Animal Behavior Processes, 1976, 2, 97-116.

Panksepp, J., Trowill, J. A. Positive and negative contrast effects with hypothalamic reward. Physiology \& Behavior, $1969,4,173-175$.

Panrge Pp, J., \& Trowill, J. A. Positive incentive contrast with rewarding electrical stimulation of the brain. Journal of Comparative and Physiological Psychology, 1970, 70, 358-363.

Pankserp, J., Trowill. J. Positive and negative contrast in licking with shifts in sucrose concentration as a function of food deprivation. Learning \& Motivation, 1971, 2, 49-57.

PAvrov, I. P. Conditioned reflexes. London: Oxford University Press, 1927.

Pizpen, W. A., \& MARx, M. H. Effects of within-session incentive contrast on instrumental acquisition and performance. Journal of Experimental Psychology, 1963, 65, 568-571.

Pert, A., \& Bitrenuan, M. E. Reward and learning in the turtle. Learning \& Motivation, 1970, 1, 121-128.

Pert, A., \& Gonzalez, R. C. Behavior of the turtle (Chrysemys 
picta picta) in simultaneous, successive, and behavioral contrast situations. Journal of Comparative and Physiological Psychology, 1974, 87, 526-538.

Peters, D. P., \& McHose, J. H. Effects of varied preshift reward magnitude on successive negative contrast effects in rats. Journal of Comparative and Physiological Psychology, 1974, 86, 85-95.

Pinel, J. P., \& Huana, E. Effects of periodic withdrawal on ethanol and saccharin selection in rats. Physiology \& Behavior, $1976,16,693-698$.

Pinel, J. P., \& Rovner, L. I. Saccharin elation effect. Bulletin of the Psychonomic Society, 1977, 9, 275-278.

Platt, J. R., \& GAy, R. A. Differential magnitude of reward conditioning as a function of predifferential reward magnitude. Journal of Experimental Psychology, 1968, 77, 393-396.

Premack, D. On some boundary conditions of contrast. In J. T. Tapp (Eds.), Reinforcement and behavior. New York: Academic Press, 1969.

Premack D., \& Hillix, W. A. Evidence for shift effects in the consummatory response. Journal of Experimental Psychology, $1962,63,284-288$.

Raymond, B., Aderman, M., \& Wolach, A. H. Incentive shifts in the goldfish. Journal of Comparative and Physiological Psychology, 1972, 78, 10-13.

Rescorla, R. A. Probability of shock in the presence and absence of CS in fear conditioning. Journal of Comparative and Physiological Psychology, 1968, 66, 1-5.

Resconla, R. A. Conditioned inhibition of fear resulting from negative CS-UCS contingencies. Journal of Comparative and Physiological Psychology, 1969, 67, 504-509.

Ridoens, A., \& GRAY, J. A. Influence of amylobarbitone on operant depression and elation effects in the rat. Psychopharmacologia, 1973, 32, 265-270.

Riley, A., \& Dunlap, W. P. Successive negative contrast as a function of deprivation condition following shifts in sucrose concentration. American Journal of Psychology, 1979, 92. 59-70.

RoBents, W. A. The effects of shifts in magnitude of reward on runway performance in immature and adult rats. Psychonomic Science, 1966, 5, 37-38.

Roberts, W. A., \& Pixley, L. The effect of chlorpromazine on the depression effect. Psychonomic Science, 1965, 3, 407-408.

Roop, S. S., \& Nation, J. R. Positive contrast following a shift from partial punishment to continuous reinforcement. Psychological Reports, 1976, 39, 934.

Rosellini, R. A., \& Terris, W. Incentive shift in the rat following training to resist punishment. Learning \& Motivation, 1975, $6,421-438$.

Roses, A. J. Incentive-shift performance as a function of magnitude of sucrose rewards. Journal of Comparative and Physiological Psychology, 1966, 62, 487-490.

Rosen, A. J., \& Ison, J. R. Runway performance following changes in sucrose rewards. Psychonomic Science, 1965, 2, 335-336.

Rosen, A. J., Glass, D. H., \& Ison, J. R. Amobarbital sodium and instrumental performance changes following reward reduction. Psychonomic Science, 1967, 9, 129-130.

Rosen, A. J., \& Tessel, R. E. Chlorpromazine, chlordiazepoxide and incentive shift performance in the rat. Journal of Comparative and Physiological Psychology, 1970, 72, 257-262.

Royce, J. R., Poley, W., \& Yeudall, L. T. Behavior-genetic analysis of mouse emotionality: I. Factor analysis. Journal of Comparative and Physiological Psychology, 1973, 83, 36-47.

Schrier, A. M. Effects of an upward shift in amount of reinforcer on runway performance of rats. Journal of Comparative and Physiological Psychology, 1967, 64, 490-492.

Sexbert, J. A. Positive and negative contrast effects as a function of shifts in percentage of reward. Bulletin of the Psychonomic Society, 1979, 13, 19-22.
Seybert, J. A., \& Mellaren, R. L. Positive contrast: Control of ceiling effect using a long runway. Psychological Reports, $1972,31,14$.

Soro, J. A., Glotfelty, R. A., \& Podlesni, J. A. Contrast effects and delay of reward in the double alleyway. Psychonomic Science, 1969, 16, 29-31.

Soro, J. A., \& Weinstock, S. Effects of delay on subsequent running under immediate reinforcement. Journal of Experimental Psychology, 1963, 66, 260-263.

Shanab, M. E. Positive transfer between delay and nonreward. Journal of Experimental Psychology, 1971, 91, 98-102.

Shanab, M. E., \& Biller, J. D. Positive contrast in the runway obtained following a shift in both delay and magnitude of reward. Learning and Motivation, 1972, 3, 179-184.

Shanab, M. E., Birnbaum, D. W., \& Cavallaro, G. Positive contrast obtained in reacquisition following interpolation of nonreinforced or partially reinforced trials. Learning and Motivation, 1974, 5, 258-271.

Shanab, M. E., \& Cavallaro, G. Positive contrast obtained in rats following a shift in schedule, delay, and magnitude of reward. Bulletin of the Psychonomic Society, 1975, 5, 109-112.

Shanab, M., Domino, J., \& Melnose, S. The effects of shifts in delay of liquid sucrose reward in thirsty rats. Bulletin of the Psychonomic Society, 1977, 10, 287-290.

Shanab, M. E., Domino, J., \& Ralph, L. The effects of repeated shifts in magnitude of food reward upon the barpress rate in the rat. Bulletin of the Psychonomic Society, 1978, 12, 29-31.

Shanab, M. E., \& Ferrell, H. J. Positive contrast in the Lashley maze under different drive conditions. Psychonomic Science, 1970, 20, 31-32.

Shanab, M. E., \& Ferrell, H. J. Transfer between downshift in reward magnitude and continuous delay of reward. Learning and Motivation, 1975, 6, 241-252.

Shanab, M. E., France, J., \& Young, T. Negative contrast effect obtained with downshifts in magnitude but not concentration of solid sucrose reward. Bulletin of the Psychonomic Society, 1975, 5, 429-432.

Shanab, M. E., France, J., \& Youna, T. Positive and negative contrast effects obtained following shifts in liquid sucrose reward in thirsty rats. Animal Learning \& Behavior, 1976, 4, 9-12.

Shanab, M. E., \& McCuistion, S. Effects of shifts in magnitude and delay of reinforcement upon runway performance in the rat. Psychonomic Science, 1970, 21, 264-266.

Shanab, M. E., Rouse, L. O., \& Cavallaro, G. Effects of shifts in delay of reward in rats as a function of reward magnitude. Journal of General Psychology, 1973, 89, 59-66.

Shanab, M. E., Sandens, R., \& Premack, D. Positive contrast in the runway obtained with delay of reward. Science, 1969 , 164, 724-725.

Shanab, M. E., \& Spencer, R. E. Positive and negative contrast effects obtained following shifts in delayed water reward. Bulletin of the Psychonomic Society, 1978, 12, 199-202.

Shanaz, M. E., \& White, R. Positive contrast obtained with punishment. Journal of General Psychology, 1972, 86, 247-251.

Shanab, M. E., Youna, T., \& France, J. Negative contrast as a function of downshifts in magnitude of sucrose concentrations in thirsty rats. Bulletin of the Psychonomic Society, 1975, 5, 381-384.

Shezиan, G. A. Running and being: The total experience. New York: Simon and Schuster, 1978.

Sinclair, J. D., Walker, S., \& Jordan, W. Behavioral and physiological changes associated with various durations of alcohol deprivation in rats. Quarterly Jourmal of Studles on Alcohol, $1973,34,744-757$.

Singer, B. F. Incentive shift in a choice situation. American Journal of Psychology, 1973, 86, 183-191.

SPEAR, N. E. Replication report: Absence of a successive contrast effect on instrumental running behavior after shift in sucrose concentration. Psychological Reports, 1965, 16, 393-394. 
Spaar, N. E., \& HiLl, W. F. Adjustment to new reward: Simultaneous and successive-contrast effects. Journal of Experimental Psychology, 1965, 70, 510-519.

Spear, N. E. Retention of reinforcer magnitude. Psychological Review, 1967, 74, 216-234.

Spear, N. E., \& Pavlik, W. B. Percentage of reinforcement and reward magnitude effects in a T-maze between and within subjects. Journal of Experimental Psychology, 1966, 71, 521-528.

Spenr, N. E., \& Spitziner, J. H. Simultaneous and successive contrast effects of reward magnitude in selective learning. Psy. chological Monographs, 1966, 00(10, Whole No. 618).

Spear, N. E., \& Spitznen, J. H. Influence of degree of training and prior reinforcer magnitude on contrast effects and resistance to extinction within S. Journal of Comparative and Physiological Psychology, 1969, 68, 427-433. (a)

Spzar, N. E., \& Spitzere, J. H. Simultaneous and successive shifts in reinforcer magnitude and influence of discrimination task. Journal of Comparative and Physiological Psychology, $1969,69,160-165$. (b)

SPEnce, K. W. Behavior theory and conditioning. New Haven: Yale University Press, 1956.

Spencer, R. E., \& Shanab, M. E. Contrast effects as a function of delay and shifts in magnitude of water reward in thirsty rats. Bulletin of the Psychonomic Society, 1979, 13, 93-96.

Stanton, M., \& Amser, A. Adjustment to reward reduction (but no negative contrast) in rats, 11,14 , and 16 days of age. Journal of Comparative and Physiological Psychology, 1980, 94, 446-458.

Stevens, S. S. Sensory scales of taste intensity. Perception \& Psychophysics, 1969, 6, 302-307.

Thomas, D. R., \& King, R. D. Stimulus generalization as a function of level of motivation. Journal of Experimental Psychology, 1959, 57, 323-328.

Tinklepauah, 0 . An experimental study of representative factors in monkeys. Journal of Comparative Psychology, 1928, 8, 197-236.

Tolman, E. C. Purposive behavior in animals and men. New York: Century, 1932.

Voozl, J. R., Mixulxa, P. J., \& Spear, N. E. Effect of interpolated extinction and level of training on the "depression effect." Journal of Experimental Psychology, 1966, 72, 51-60.

Vogel, J. R., Mixulka, P. J., \& Spear, N. E. Effects of shifts in sucrose and saccharin concentrations on licking behavior in the rat. Journal of Comparative and Physiological Psychology, $1968,66,661-666$.

Vooel, J. R., \& Principi, K. Effects of chlordiazepoxide on depressed performance after reward reduction. Psychopharmacologia, 1971, 21, 8-12.
Whaner, A. R. Effects of amount and percentage of reinforcement and number of acquisition trials on conditioning and extinction. Journal of Experimental Psychology, 1961, 62 , $234-242$.

WAGNen, A. R. Frustrative nonreward: A variety of punishment? In B. A. Campbell \& R. M. Church (Eds.), Punishment. New York: Appleton-Century-Crofts, 1969.

Walkenbach, J., Haddad, N. F., \& Mellaren, R. L. N-length shifts and successive contrast effects. Bulletin of the Psychonomic Society, 1978, 11, 83-85.

Wayner, M. J., Greenbero, I., Tartaglione, D., Nolley, D., Fraley, S., \& Cotr, A. A new factor affecting the consumption of ethyl alcohol and other sapid fluids. Physiology \& Behavior, 1972, 8, 345-362.

WEIN⿴囗E IN, L. Negative incentive contrast effects with saccharin vs sucrose and partial reinforcement. Psychonomic Science, 1970, 21, 276-278. (a)

Weingtein, L. Negative incentive contrast with sucrose. Psychonomic Science, 1970, 19, 13-14. (b)

Weinstein, L. Effects of magnitude of reward increment on positive incentive contrast effects in the rat. Bulletin of the Psychonomic Society, 1977, 9, 233-235.

Weinstock, R. B. Preacquisition exploration of the runway in the determination of contrast effects in the rat. Journal of Comparative and Physiological Psychology, 1971, 75, 107-115.

Wolach, A. H., \& LATTA, K. Reward magnitude shifts in turtles (Pseudemys scripta elegans). Psychological Record, 1974, 24, 237-241.

Wolach, A. H., Raymond, B., \& HuRst, J. W. Reward magnitude shifts with goldfish. Psychological Record, 1973, 23, 371-376.

Woods, P. J. Performance changes in escape conditioning following shifts in the magnitude of reinforcement. Journal of Experimental Psychology, 1967, 75, 487-491.

Woodworth, R. S., \& Schlosbero, H. Experimental psychology. New York: Holt, Rinehart, and Winston, 1954.

Zenman, D. Response latency as a function of the amount of reinforcement. Journal of Experimental Psychology, 1949, 39, 466-483.

\section{NOTE}

1. Some written comments concerning the relationship between behavioral contrast and discrete-trial incentive contrast are available from the author.

(Manuscript received February 22, 1982; revision accepted for publication September 21, 1982.) 